\title{
La genèse et la manifestation des structures d'un point de vue cognitiviste et (post-)structuraliste
}

\section{Sébastien Miravete}

\section{(2) OpenEdition}

\section{Journals}

\section{Electronic version}

URL: http://journals.openedition.org/appareil/3005

DOI: 10.4000/appareil.3005

ISSN: 2101-0714

\section{Publisher}

MSH Paris Nord

\section{Electronic reference}

Sébastien Miravete, "La genèse et la manifestation des structures d'un point de vue cognitiviste et (post-)structuraliste", Appareil [Online], Articles, Online since 07 February 2019, connection on 30 January 2021. URL: http://journals.openedition.org/appareil/3005 ; DOI: https://doi.org/10.4000/ appareil.3005

This text was automatically generated on 30 January 2021.

\section{$\Theta \oplus \Theta \Theta$}

Appareil est mis à disposition selon les termes de la Licence Creative Commons Attribution - Pas d'Utilisation Commerciale - Pas de Modification 4.0 International. 


\title{
La genèse et la manifestation des structures d'un point de vue cognitiviste et (post-)structuraliste
}

\author{
Sébastien Miravete
}

\section{Le structuralisme classique}

\subsection{Le structuralisme linguistique}

1 Une «structure » est le nom que l'on donne traditionnellement en sciences humaines, depuis les travaux du cercle de Prague (Troubetzkoy, Jakobson) et de Lévi-Strauss, à un ensemble de relations invariantes entre les éléments de plusieurs systèmes : les éléments d'un premier système ne ressemblent pas aux éléments d'un second système, mais ils entretiennent entre eux les mêmes relations. L'analyse structurale consiste à se donner un grand nombre de systèmes composés d'éléments différents et à chercher les relations régissant le fonctionnement de n'importe quel système. Ces relations forment une structure à partir du moment où elles sont les mêmes quel que soit le système et permettent d'expliquer le comportement des éléments dans chaque système. Cette méthode n'est pas toujours celle qu'on utilise en sciences dans un premier temps. On peut se contenter de comparer, pour commencer, les éléments de plusieurs systèmes afin de comprendre leur organisation. On opère alors une classification: les sons [m], [n], [n], [y] présents dans plusieurs langues se ressemblent dans la mesure où ils sont générés de la même façon par l'appareil phonatoire (le son s'échappe par le nez grâce à l'abaissement du palais); ils partagent ainsi une sonorité et un mode de production commun et c'est pourquoi ils appartiennent à la classe des « consonnes nasales ». Ces classifications sont sans aucun doute indispensables et donnent un premier aperçu de la façon dont l'appareil phonatoire produit des sons. On peut ainsi avoir l'impression que la classification est une méthode capable d'expliquer à elle seule le fonctionnement de plusieurs systèmes. Elle demeure néanmoins insuffisante pour comprendre la production des sons d'une langue. 
2 La linguistique a en effet été profondément bouleversée au $\mathrm{xx}^{\mathrm{e}}$ siècle par la découverte de structures régissant toutes les langues. Cette "science sociale ${ }^{1}$ " a clairement montré que le mécanisme de l'appareil phonatoire ne suffit pas à lui seul à expliquer la production des sons-éléments composant une langue. Un son est tout d'abord un phonème s'il modifie le sens d'un mot; " $[\mathrm{m}]$ » est un phonème parce que son remplacement dans le mot «âme» par «[n] » modifie la signification de ce mot ("âme » devient " âne»). On observe, en d'autres termes, une analogie: " $[\mathrm{m}]$ » est à "âme» ce que "[n]" est à "âne", à savoir un son susceptible de modifier la signification de ce mot si on le remplace. Certes, les phonèmes sont classés dans la même catégorie "phonème" parce qu'ils ont une propriété commune: leur remplacement altère la signification d'un mot. Cependant, cette propriété n'a de sens que si l'on conserve les différences entre les sons et les significations. Elle n'exprime par conséquent que des relations invariantes entre les éléments de plusieurs systèmes $([\mathrm{m}] /$ âme $=[\mathrm{n}] / \mathrm{âne})$ et non des ressemblances entre ces éléments $([\mathrm{m}]=[\mathrm{n}]$, « âme » $=$ " âne »). On n'obtient donc pas la liste des phonèmes en décomposant un mot en sons élémentaires: le linguiste ne se contente pas d'exhiber une caractéristique physique commune (sonorité ou mode de production identique) à un ensemble de sons et de recomposer les mots à partir de ces sons de base (les consonnes nasales, etc.). En d'autres termes, la linguistique ne se réduit pas à une perspective purement physiologique, fondée sur la seule observation du fonctionnement de l'appareil phonatoire ${ }^{2}$. On cherche plutôt à substituer certains sons à d'autres et on ne retient que ceux susceptibles de produire une nouvelle signification. On ne découvre donc les phonèmes qu'en dévoilant les relations invariantes qu'entretiennent les sons (ou leurs modes de production) et les significations : « $[\mathrm{m}]$ » et «âme » (système 1 ) et « $[\mathrm{n}]$ » et " âne » (système 2) entretiennent la même relation (de changement de signification), parce que dans les deux cas la modification de « $[\mathrm{m}]$ » ou de « $[\mathrm{n}]$ » altère le sens du mot. Saussure n'employait pas encore le terme de «structure». Il est évident néanmoins que la méthode employée par celui-ci pour mettre au jour les phonèmes est de nature structurale, puisqu'elle consiste à dévoiler certaines analogies, c'est-à-dire certaines relations invariantes ${ }^{3}$.

3 Ces dernières ne sont pas les seules observables ni les plus décisives. Les linguistes ne vont prendre pleinement conscience de l'intérêt de l'approche structurale qu'à partir du moment où ils vont découvrir, grâce aux travaux de l'école russe, les véritables relations régissant la production des phonèmes. On ne passe pas du mot « âme » au mot «âne » en substituant simplement au son [m] un son [n] quelconque. Le son [n] n'est pas arbitrairement choisi parmi l'ensemble des sons susceptibles d'être produits par l'appareil phonatoire. La palette des sons disponibles est en effet infinie. Pourquoi choisir le son [n]? Il n'existe effectivement qu'un nombre très limité de phonèmes susceptibles de se substituer les uns aux autres dans une langue, et cela ne peut pas s'expliquer par les limites de l'appareil phonatoire. Cette question demeure sans réponse tant qu'on limite l'analyse structurale aux relations entre les sons et les significations. Il faut étendre son usage et s'intéresser aux relations entre les phonèmes eux-mêmes. C'est ainsi que la portée et l'importance de cette méthode se révèlent.

4 Le passage d'un phonème à un autre consiste en effet à passer au moins d'un « trait » à son opposé. Le passage du trait «grave » au trait «aigu» rend compte du passage de [m] à [n] et ainsi du mot «âme » au mot « âne " ». À titre d'illustration, on peut aussi obtenir [l] à partir de [m] en passant, entre autres, du non-vocalique au vocalique, ou 
encore [a] à partir de [m] en passant du non-vocalique au vocalique et du consonantique au non-consonantique. Ces traits distinctifs (grave/aigu, vocalique/nonvocalique, consonantique/non-consonantique, etc.) paraissent peu nombreux dans le monde (une douzaine ${ }^{5}$ ) et limitent le nombre de phonèmes susceptibles de différer d'un autre - par exemple de $[\mathrm{m}]$. C'est pourquoi, le nombre de phonèmes que compte une langue est relativement limité (quelques dizaines). Ces relations d'opposition restent les véritables productrices des phonèmes d'une langue - ces derniers n'étant donc pas entièrement choisis au hasard. Elles constituent la structure phonologique d'une langue (Jakobson) - celle-ci pouvant dès lors être définie par l'ensemble des traits distinctifs qu'elle possède.

5 La découverte d'un trait distinctif repose une nouvelle fois sur une analyse structurale dont le rôle est de révéler certaines analogies : « an » est à « a » ce que « on » est à « 0 " (trait nasal/non nasal), etc. La classification des phonèmes en fonction de leur mode de production permet d'identifier certaines ressemblances (nasales, vocaliques, etc.). Elle s'avère indispensable, mais insuffisante pour percevoir la structure, c'est-à-dire dans ce cas précis les couples d'oppositions entre les phonèmes (les traits distinctifs : nasal/non nasal; vocalique/non vocalique, etc.). La classification fournit un matériau précieux (des modes de production semblables) que le chercheur peut exploiter: plus précisément, la classification lui fournit un ensemble de traits (nasal, vocalique). Il lui suffit alors d'exhiber les relations d'opposition qu'entretiennent ces traits (nasal/non nasal, etc.) et qui expliquent la formation des phonèmes. La méthode structurale ne consiste donc pas à exclure la classification, mais à lui conférer une fonction spécifique et primitive. Seule une approche classificatoire réductionniste, rejetant dans un second temps toute forme d'analyse structurale, demeurerait dans l'impossibilité d'expliquer la formation des phonèmes.

\subsection{Le structuralisme ethnologique}

6 L'ethnologie va elle aussi réaliser ses plus grandes découvertes par la mise au jour de certaines relations invariantes entre les membres de différentes sociétés ${ }^{6}$. C'est ce qu'on appelle communément le tournant « structuraliste » de l'ethnologie. On se donne d'abord un ensemble de composants de bases : père, mère, fils, oncle (frère de la mère). Puis, on se donne un ensemble de relations possibles (autorité, tendresse) ritualisées (obligatoires et respectant un certain protocole) entre ces éléments. Ces éléments et ces relations sont naturellement identifiés au moyen d'une méthode classificatoire : toutes les sociétés possèdent des éléments et des relations semblables - la classe des " pères ", des «mères ", des « relations ritualisées de tendresse », etc.

On ne cherche pas à calculer l'ensemble des relations possibles entre ces composants. La structure, au sens de Lévi-Strauss, se révèle lorsqu'on observe, dans de multiples sociétés, le caractère antithétique de certaines relations ritualisées: si le père $(p)$ entretient une relation d'autorité ritualisée (a) avec le fils (f), alors l'oncle (o) entretient nécessairement une relation de tendresse ritualisée ( $\mathrm{t}$ ) avec le fils (f); si l'oncle entretient une relation d'autorité ritualisée avec le fils ( $\mathrm{a} f$ ), alors le père entretient nécessairement une relation de tendresse ritualisée avec le fils $(\mathrm{pt} f$ ). La relation entre l'oncle et le fils est par conséquent toujours l'opposé de celle qui existe entre le père et le fils, que l'on soit dans une société patrilinéaire (où le fils hérite du père et entretient avec lui une relation ritualisée d'autorité) ou dans une société matrilinéaire (où le fils 
hérite généralement de l'oncle et entretient avec lui une relation ritualisée d'autorité). On peut donc écrire : « $p$ a f/o $t \mathrm{f}=\mathrm{pt} f / \mathrm{o}$ a $\mathrm{f}$ ». On observe autrement dit une certaine analogie : la relation père-fils dans une société patrilinéaire est à la relation oncle-fils d'une société patrilinéaire ce que la relation père-fils dans une société matrilinéaire est à la relation oncle-fils d'une société matrilinéaire, à savoir son opposé. Pourquoi cette structure relativement simple, découverte par Radcliffe-Brown ${ }^{7}$, avait-elle échappé auparavant aux travaux des ethnologues?

Ces derniers ne comprenaient pas, jusqu'à l'emploi de la méthode structurale, l'existence d'une relation ritualisée entre l'oncle et le fils dans une société patrilinéaire. Certains y voyaient la persistance d'une relation ritualisée issue d'un modèle matrilinéaire antérieur8. Ils étaient ainsi contraints de supposer, sans aucune preuve, que toute société patrilinéaire avait été matrilinéaire. Ils rapprochaient ainsi abusivement la relation ritualisée entre l'oncle et le fils (d'autorité) d'une société matrilinéaire de celle que l'on trouve dans une société patrilinéaire (de tendresse). La relation de tendresse était en effet à leurs yeux le résidu d'une relation d'autorité, c'està-dire une sorte de relation d'autorité affaiblie. Ils percevaient entre la relation d'autorité et celle de tendresse une certaine ressemblance. Ils ne retenaient de la relation d'autorité que le trait commun qu'elle partage avec la relation de tendresse, à savoir le fait d'être ritualisée. Cette propriété commune voilait l'incommensurabilité des deux relations et surtout leur caractère antithétique. De toute évidence, la tendresse n'a strictement rien à voir avec l'autorité. L'ancienne ethnologie employait une méthode d'investigation exclusivement classificatoire. Cette dernière la conduisait à se focaliser à tort sur une ressemblance grossière entre « o a f » et « o $t \mathrm{f}$ » et à faire de la seconde le résidu historique de la première. En vérité, toutes ces relations élémentaires différent profondément ( $\mathrm{p}$ a $\mathrm{f} \neq \mathrm{p} \mathrm{t} f \neq \mathrm{o}$ a $\mathrm{f} \neq \mathrm{o} \mathrm{t} \mathrm{f}$ ). Entre une société matrilinéaire et patrilinéaire, seules certaines relations sont invariantes ( $p$ a f/o $t \mathrm{f}=\mathrm{pt}$ $\mathrm{f} / \mathrm{o}$ a f). Cette structure ne se dévoile que lorsqu'on renonce à faire de la classification une méthode capable à elle seule de mettre au jour les relations susceptibles d'expliquer l'organisation familiale et ritualisée d'une société. L'analyse structurale recourt à la classification pour identifier des éléments (le père, l'oncle, le fils) et des relations ritualisées communes (tendresse, autorité) entre diverses sociétés. À l'instar de la linguistique, elle ne peut se contenter de ce travail primitif de prospection. Sinon, elle demeure dans l'incapacité de mettre au jour les relations antithétiques régissant le fonctionnement des systèmes de parenté dans ces sociétés.

\subsection{La nature paradoxale du structuralisme}

Une méthode d'explication exclusivement fondée sur la classification n'est pas le seul obstacle à la compréhension des phénomènes. Il ne suffit pas d'observer la manière dont un son est produit par l'appareil phonatoire pour saisir son mécanisme de formation. Il est vrai qu'on peut dans un premier temps observer les relations de cause à effet entre les différents éléments d'un seul système. Un observateur note que l'action de tel élément entraîne la réaction de tel autre : le déplacement d'un pendule actionne régulièrement une roue dentée dans une horloge mécanique; la contraction du cœur assure la circulation du sang dans un organisme; l'oncle manifeste au fils de sa sœur une relation de tendresse par l'intermédiaire de tel rituel; un pays manque de certaines ressources et décide pour cette raison d'en envahir un autre; etc. Toutes ces interactions entre les éléments d'un système peuvent être consignées. Elles donnent 
l'impression que les éléments influent les uns sur les autres comme les pièces d'un mécanisme ou les événements d'un récit. On explique uniquement un système physique, biologique ou social à partir des interactions qu'on observe entre ses éléments ou entre ses éléments et ceux d'un autre système. Cela renvoie en dernière instance à une conception que nous qualifierons $d^{\prime}$ '«interactionnaliste" de l'explication: on dissèque un ou plusieurs systèmes et on saisit ensuite les relations qu'entretiennent leurs éléments leurs interactions (chocs, attirances, etc.), la fonction des uns par rapport aux autres, le résultat final de leurs interactions, etc.

10 Jakobson et Lévi-Strauss montrent et rappellent chacun à leur manière à quel point une telle conception de la relation entre les éléments ou entre les systèmes demeure insuffisante - même si elle reste nécessaire ${ }^{9}$. On ne peut pas comprendre le fonctionnement d'un système sans observer en parallèle d'autres systèmes et chercher entre eux des relations invariantes, c'est-à-dire des structures communes. Certes, on peut être séduit dans un premier temps par une explication essentiellement fondée sur les interactions observées entre les éléments d'un ou plusieurs systèmes. Des événements entraînent d'autres événements. On relève des cascades d'effets. On peut même avoir le sentiment de décrire ainsi une histoire avec précision et de respecter la manière singulière dont les événements s'y enchainent. Mais ces descriptions subtiles et détaillées nous induisent en erreur ou nous maintiennent dans l'obscurité : l'appareil phonatoire semble être à lui seul la cause de la formation des phonèmes d'une langue où la quantité limitée de phonèmes demeure un mystère; la relation ritualisée de tendresse entre l'oncle et le fils dans une société patriarcale devient le résidu d'une société matriarcale. Il ne s'agit pas de critiquer une discipline en tant que telle, mais de dénoncer une conception exclusivement interactionnaliste du récit, de l'histoire, de l'ethnologie, etc. Philippe Descola le souligne dans un entretien récent : le point de vue interactionnaliste sous sa forme réductionniste exige d'être dépassé ${ }^{10}$. Sinon, les causes des phénomènes risquent de ne pas être celles que nous croyons observer. Il importe de ne pas confondre les relations interactionnelles (diverses et illusoirement explicatives) que l'on peut relever entre les éléments d'un seul système (ou entre les éléments d'un système et de quelques autres systèmes situés autour de lui) et les relations structurales (invariantes et explicatives) que l'on constate entre les éléments de nombreux systèmes très différents en apparence. Tel est l'enseignement que tirent Jakobson et LéviStrauss : les causes profondes des interactions entre les éléments d'un système particulier ou singulier ne se révèlent paradoxalement qu'à travers un certain point de vue plus global capable de saisir certaines relations invariantes entre les éléments de plusieurs systèmes. Le «structuralisme » est devenu selon nous le nom de ce point de vue paradoxal, durant la seconde moitié du $\mathrm{xx}^{\mathrm{e}}$ siècle, dans l'histoire des sciences ${ }^{11}$.

\subsection{L'origine du structuralisme}

11 L'analyse structurale permet effectivement de mettre au jour des causes insoupçonnées et impossibles à découvrir sans elle : le mode de production des phonèmes ; l'existence d'une relation de tendresse entre l'oncle et le fils dans une société patriarcale, etc. La simple observation des interactions entre les éléments d'un ou plusieurs systèmes ne suffit jamais. On pourrait multiplier les exemples. Sur Terre, un litre d'eau pèse environ 1 kilogramme alors qu'il pèserait approximativement 380 grammes sur Mercure et 2,5 kilogrammes sur Jupiter. De telles différences pour un même objet resteraient incompréhensibles si on ne révélait pas la relation invariante qu'entretiennent le poids 
et la pesanteur d'une planète. Le poids (p) est toujours égal à la masse de l'objet $(\mathrm{m})$ multipliée par la pesanteur $(\mathrm{g}):\langle\mathrm{p}=\mathrm{m} \times \mathrm{g} \|$. En ce sens, le poids d'un objet et la pesanteur ne cessent de varier en fonction de la planète sur laquelle on se situe chacune ayant une pesanteur plus ou moins forte. Il n'en demeure pas moins que la relation entre le poids et la pesanteur reste identique. L'équation $" \mathrm{p}=\mathrm{m} \times \mathrm{g}$ » exprime l'invariance de cette relation, au même titre que n'importe quelle équation. Les lois fondamentales de la physique ne sont rien d'autre, en ce sens, que des analogies exprimées à l'aide d'équations fondamentales: la valeur de la variable $\mathrm{x}_{1}$ est à celle de $\mathrm{x}_{2}$ et à celle de $\mathrm{x}_{3}$ et à celle de $\mathrm{x}_{4}$, etc., dans tel système ce que la valeur de la variable $\mathrm{x}_{1}$ est à celle de $\mathrm{x}_{2}$ et à celle de $\mathrm{x}_{3}$ et à celle de $\mathrm{x}_{4}$, etc. dans n'importe quel autre système les variables n'ayant pas le plus souvent les mêmes valeurs dans ces systèmes. Le poids d'un litre d'eau $\left(\mathrm{x}_{1}=1 \mathrm{~kg}\right)$ est par exemple à la pesanteur sur Terre $\left(\mathrm{x}_{2}=9,8\right)$ ce que ce poids $\left(\mathrm{x}_{1}=380 \mathrm{~g}\right)$ est à la pesanteur sur Mercure $\left(\mathrm{x}_{2}=3,7\right)$ - la relation invariante peut être exprimée à l'aide d'un simple rapport ( $\mathrm{p} / \mathrm{g}=\mathrm{m} »)$ comme nous l'avons fait jusqu'à présent ( $[\mathrm{m}] / \mathrm{a} m e=[\mathrm{n}] /$ âne $» ; " \mathrm{p}$ a f/o t $\mathrm{f}=\mathrm{ptf} / \mathrm{o}$ a f»), mais ce n'est évidemment pas toujours le cas dans des équations ou des structures plus complexes. Depuis le $\mathrm{XVII}^{\mathrm{e}}$ siècle, les sciences physiques révèlent en définitive les structures régissant le comportement des objets matériels. Le structuralisme est par conséquent une méthode qui trouve en dernière instance sa racine dans la physique classique.

On pourrait objecter que les modélisations employées par Lévi-Strauss et Jakobson sont purement qualitatives. Elles ne décrivent en effet que des oppositions entre des relations (la tendresse est l'opposé de l'autorité) ou des éléments ([m] est l'opposé de [n] selon le trait distinctif grave/aigu). On ne trouve pas dans ces modèles la présence de grandeurs (l'intensité, l'énergie, la masse, la température, le volume, etc.), c'est-àdire d'entités susceptibles d'être " plus ou moins grandes » et désignées le plus souvent par une variable quantitative - les variables « $\mathrm{p}$ », « $\mathrm{m}$ » et « $\mathrm{g}$ » dans l'équation « $\mathrm{p}=\mathrm{m}$ $\times g »$. Or, les sciences physiques dévoilent généralement des relations invariantes de nature quantitative : les équations fondamentales ne relient que des grandeurs entre elles. On pourrait croire par conséquent que le structuralisme en sciences humaines rompt avec le structuralisme issu des sciences physiques.

Une telle perspective négligerait, cependant, le fait que la structure chez Lévi-Strauss, comme chez Jakobson, s'exprime au moyen d'un langage « symbolique », «formel » ou « logique ${ }^{12}$ »: on se donne des symboles-éléments (p, m, o, f) et des symboles-relations $(a, t)$; la structure n'est rien d'autre que l'ensemble des relations qu'elle contraint ou interdit entre ces symboles (si « $p$ a $f$ » alors « o $t$ » est contraint et «o a $f$ » interdit). On peut ainsi prédire l'ensemble des relations symboliques autorisées par la structure (l'ensemble des combinaisons possibles) et ainsi l'impact de la modification d'un élément sur les autres. Ces langages qualitatifs sont tout aussi prédictifs et précis que des langages quantitatifs. De tels langages sont en outre aujourd'hui à la base de toutes les mathématiques (théorie des ensembles, théorie des catégories) et de l'informatique. Il serait difficile, pour cette raison, de ne pas percevoir dans le structuralisme en sciences humaines tel qu'on le trouve chez Jakobson et Lévi-Strauss, une extension des méthodes empiriques (saisir par l'observation et l'expérimentation des identités de rapport) et mathématiques (expression d'une identité de rapport au moyen d'une équation ou d'un langage symbolique) apparues au xvII siècle dans les sciences physiques - c'est pourquoi nous parlerons de structuralisme « classique ». 
Les sciences humaines ne mobiliseraient pas toutefois l'analyse structurale si cette dernière ne permettait pas de résoudre certains problèmes demeurés insolubles sans elle : pourquoi les langues ont-elles si peu de phonèmes alors que l'appareil phonatoire est capable d'en produire une multitude ? Pourquoi observe-t-on une relation ritualisée entre l'oncle et le fils dans une société patrilinéaire? Le structuralisme classique ne consiste pas dans l'application aveugle d'une méthode issue des sciences physiques. Il «montre » des systèmes " concrets » et " met en évidence » leurs structures, comme le souligne Troubetzkoy dans un texte devenu célèbre ${ }^{13}$. Chaque problème soulevé par tel ou tel ensemble de données empiriques est l'occasion de réévaluer la pertinence de l'analyse structurale. Le structuralisme classique est toujours en ce sens en train de se faire. Il ne se développe qu'au contact des faits.

\section{Repenser les structures}

\subsection{Une nouvelle structure paradigmatique pour penser la manifestation des structures en sciences humaines et sociales : le conditionnement}

Quelles difficultés le structuralisme sous sa forme classique, c'est-à-dire tel que nous venons de le présenter, rencontre-t-il ? Dans quelle mesure exige-t-il une importante refonte? L'exemple du conditionnement se révèle sur ce point particulièrement instructif. On peut contraindre les êtres vivants à adopter certaines attitudes. Le "béhaviorisme" ou si l'on préfère le "comportementalisme " semble avoir mis en lumière depuis longtemps et implicitement la structure classique d'un tel conditionnement. Si on présente un morceau de sucre à un chien (stimulus), celui-ci se met à saliver (réponse). Si on associe régulièrement à ce stimulus un autre stimulus (le son émis par un sifflet), alors on peut finir par déclencher la réponse en l'absence même du morceau de sucre : le chien salive dès qu'il entend un coup de sifflet (Pavlov). On dit alors que le chien a été conditionné à répondre au son du sifflet. On peut dès lors définir la structure classique du conditionnement répondant (Watson) de la façon suivante: quel que soit le stimulus associé (le coup de sifflet, le son émis par un diapason, etc.) et quelle que soit la relation de cause à effet déjà présente entre un stimulus et sa réponse (saliver à la vue d'un morceau de sucre), alors une relation de cause à effet (notée « $\rightarrow$ ) entre le stimulus et la réponse s'établit. Autrement dit, quel que soit le stimulus associé « $\mathrm{S}_{\mathrm{An}}$ " $\left(\mathrm{S}_{\mathrm{A} 1}, \mathrm{~S}_{\mathrm{A} 2}\right.$, etc.) et quelle que soit la relation « $\mathrm{S}_{\mathrm{m}} \rightarrow$ $\mathrm{R}_{\mathrm{m}}$ » (« $\mathrm{S}_{1} \rightarrow \mathrm{R}_{1}$ », « $\mathrm{S}_{2} \rightarrow \mathrm{R}_{2}$ », etc.), alors la relation « $\mathrm{S}_{\mathrm{An}} \rightarrow \mathrm{R}_{\mathrm{m}}$ » est vérifiée : « $\mathrm{S}_{\mathrm{A} 1} /\left(\mathrm{S}_{1} \rightarrow\right.$ $\left.\mathrm{R}_{1}\right)=\mathrm{S}_{\mathrm{A} 2} /\left(\mathrm{S}_{1} \rightarrow \mathrm{R}_{1}\right)=\mathrm{S}_{\mathrm{A} 1} /\left(\mathrm{S}_{2} \rightarrow \mathrm{R}_{2}\right)=\mathrm{S}_{\mathrm{A} 2} /\left(\mathrm{S}_{2} \rightarrow \mathrm{R}_{2}\right)=\ldots=\mathrm{S}_{\mathrm{A}} \rightarrow \mathrm{R} »$. Pour le dire autrement, quel que soit $n$, quel que soit $m$, « $S_{A n} /\left(S_{m} \rightarrow R_{m}\right)=S_{A n} \rightarrow R_{m}$ ".

16 Cette analyse à la fois structurale et béhavioriste dissimule toutefois une importante difficulté. Un rat peut finir par comprendre qu'actionner (réponse) un levier (stimulus) lui permet d'obtenir une boulette de nourriture (conséquence). Le conditionnement dit " opérant » (Skinner) s'appuie par conséquent moins sur une relation de base « $\mathrm{S} \rightarrow \mathrm{R}$ » (la vue du morceau de sucre entraîne la salivation) que sur une conséquence " $C$ » (le gain de nourriture) pour engendrer le comportement souhaité. Il importe cependant que cette conséquence entraîne une réponse chez le rat : la nourriture fait envie au rat, au même titre que le morceau de sucre fait saliver le chien. « $C$ » n'est donc rien d'autre qu'une relation $« S \rightarrow R$ » dans laquelle le « $R »$ reste à déterminer: le rat ne sait pas 
encore quelle réponse adopter pour obtenir la nourriture. De la même manière, le son associé dans l'expérience de Pavlov ne peut finir par déclencher la réponse souhaitée que si ce stimulus est traité par le chien comme un élément susceptible d'entraîner une réponse. On peut observer une relation de cause à effet entre un stimulus (une boîte de yaourt) et une réponse (étiqueter la boîte) dans le fonctionnement d'une machine automatique. On ne parviendra jamais à apprendre à cette machine à réagir à un stimulus associé (il ne servira à rien de siffler chaque fois qu'une boîte de yaourt se présentera) parce que cette machine est incapable de bâtir un lien du type « stimulus $\rightarrow$ réponse ». Le conditionnement répondant dissimule lui aussi une relation « $S \rightarrow R$ » au sein de " $S_{A}$ ": $S$ est déjà le membre d'un couple « $S \rightarrow R$ » pour le chien, même s'il ne sait pas encore exactement à quel « $R$ » l'associer.

C'est pourquoi la structure du conditionnement échappe selon nous au béhaviorisme. Ce dernier suppose en effet implicitement l'existence d'un système cognitif (même s'il le rejette explicitement) capable de traiter un stimulus comme une « information », c'est-àdire comme un élément susceptible de le renseigner sur la réponse à adopter ${ }^{14}$. Dans une telle perspective, il n'existe que des couples « $S \rightarrow R »$ ( $S$ » n'existe jamais seul), même lorsque la réponse « $\mathrm{R}$ » n'est pas connue par le système cognitif : un stimulus est toujours perçu comme une source d'information plus ou moins pertinente. La structure du conditionnement peut dès lors être réinterprétée dans le cadre d'une analyse à la fois structurale (recourt à la méthode analogique) et cognitiviste (remplacement de la notion de stimulus par celle d'information). Sa formulation se révèle même plus simple : si une information «I " (un coup de sifflet ; actionner le bras du levier) dont on ne connaît pas encore l'importance, entretient une relation régulière et de contiguïté avec à une autre « Iimp » dont on connaît l'importance (morceau de sucre, boulette), alors celle-ci peut devenir une information prédictive importante (si le coup de sifflet est produit, alors le chien peut espérer obtenir un morceau de sucre; si le rat appuie sur le levier, alors il peut espérer obtenir des boulettes). En d'autres termes, quel que soit $n$ et quel que soit $m, \ll \mathrm{I}_{\mathrm{n}} / \operatorname{Iimp}_{\mathrm{m}}=\mathrm{I}_{\mathrm{n}} \rightarrow \operatorname{Iimp}_{\mathrm{m}} »$.

18 Cela est encore plus évident lorsqu'on place des pigeons dans une cage munie d'un dispositif automatique de distribution de nourriture ${ }^{15}$. Les pigeons adoptent alors progressivement certains comportements arbitraires, mais susceptibles à leurs yeux de provoquer l'arrivée de la nourriture. Ils développent selon skinner des "superstitions»: ils croient qu'une attitude spécifique a un impact ou au moins favorise l'apparition de la nourriture. Il compense donc l'absence d'information prédictive (l'absence d'un coup de sifflet ou d'un levier) par la création d'une information prédictive (incarnée dans une action quelconque). Ils s'auto-conditionnent.

Cela suggère une nouvelle fois que les pigeons souhaitent établir des relations de cause à effet. Ils aspirent à construire des connaissances et à tisser des relations entre des informations fondamentales (ce stimulus satisfait tel besoin vital) et des informations susceptibles d'annoncer ou de provoquer la venue de ces informations fondamentales. Le conditionnement n'a donc lieu que si l'information prédite par une autre revêt aux yeux de certains animaux (humains ou non humains) une importance vitale ou capitale, et la structure du conditionnement est in fine : quel que soit $n$ et quel que soit $m$, il doit exister un $\mathrm{I}_{\mathrm{n}}$ tel que " $\mathrm{I}_{\mathrm{n}} / \operatorname{Iimp}_{\mathrm{m}}=\mathrm{I}_{\mathrm{n}} \rightarrow \operatorname{Iimp}_{\mathrm{m}}$ ", avec " / " désignant une relation régulière de proximité spatiale et temporelle.

20 C'est parce qu'il doit y avoir une cause (« il doit exister un $I_{n}$ ») susceptible de satisfaire les besoins ou les aspirations les plus fondamentales des êtres vivants que ces derniers 
cherchent (s'ils sont dotés d'un système cognitif) à se conditionner. Le conditionnement est moins une réaction réflexe, comme on pourrait le croire dans une perspective béhavioriste, qu'une authentique structure d'apprentissage. Ce n'est pas l'expérimentateur ou encore le publicitaire qui conditionne en dernière instance l'être vivant. C'est l'être vivant qui cherche à se conditionner et qui demeure l'auteur du conditionnement - au sens où rien d'extérieur à lui ne le soumet à une telle activité. Le publicitaire ne fait que détourner à son propre profit cette structure innée et autonome d'apprentissage. Il ne la crée pas. Il ne l'actionne pas. Il ne la manipule pas comme il tirerait les ficelles d'une marionnette. Nous dirons qu'il «l'instrumentalise ${ }^{16}$ ». Comment y parvient-il ? Et pourquoi cela nous invite-t-il à revisiter le concept de structure?

21 Dans une structure linguistique, la relation "grave/aigu " permet de passer d'un phonème à un autre (par exemple de $[\mathrm{n}] \mathrm{à}[\mathrm{m}])$. Cependant, elle ne permet pas de relier un phonème à n'importe quel autre: $[\mathrm{m}]$ ne peut être relié qu'à $[\mathrm{n}]$. La relation de proximité spatio-temporelle est, quant à elle, de nature cognitive: elle consiste à élaborer une relation entre deux informations, entre l'événement « Iimp » désiré et une autre. Or, une telle relation n'est pas aussi limitée que celle que l'on trouve entre deux phonèmes. On observe en effet que les pigeons adoptent des postures diverses : à partir d'un «Iimp » quelconque (obtenir des boulettes, etc.), il peut sélectionner une posture quelconque; il n'est pas cantonné à choisir telle ou telle posture. Autrement dit, la relation dans une structure linguistique ne peut associer à un terme qu'un unique terme, alors que la relation dans une structure cognitive semblable à celle du conditionnement peut associer à un terme une multiplicité quasi infinie de termes. Certes, le choix de ces termes n'est pas arbitraire. Il obéit à certaines contraintes: le pigeon doit effectivement avoir l'impression que telle posture est à l'origine de l'arrivée de nourriture. Mais de telles contraintes laissent au pigeon une marge de manœuvre relativement importante. Elles laissent à chaque pigeon la possibilité d'inventer sa propre superstition, c'est-à-dire sa propre posture.

Il n'existe donc qu'une seule manière de forcer une structure cognitive comme celle du conditionnement à faire toujours le même choix : il faut réduire artificiellement toutes les options de l'animal à une seule: le coup de sifflet est le seul stimulus régulièrement associé au morceau de sucre; on ne présente qu'un levier au rat susceptible de lui apporter la nourriture dont il a besoin. Dans ces deux situations, la relation de proximité spatio-temporelle ne trouve qu'une information (le coup de sifflet, le levier) susceptible de produire l'événement désiré (l'arrivée de nourriture). Les expériences sur le conditionnement répondant (le chien) ou opérant (le rat, le pigeon) ne suggèrent donc pas que le conditionnement est un automatisme qui associe mécaniquement l'événement le plus régulier et le plus proche à un autre. Elles nous invitent plutôt à percevoir dans le conditionnement une activité cognitive qui s'interroge sur les informations les plus proches dans l'espace et dans le temps susceptibles de la renseigner sur la manière de satisfaire tel ou tel objectif. L'expérimentateur, mais aussi un publicitaire ou un propagandiste dans le cas d'un être humain, peut plus ou moins contrôler cette structure en lui imposant un nombre limité d'informations disponibles.

Une analyse structurale peut donc mettre au jour des relations invariantes plus ou moins limitées. Elle n'est donc pas tenue de saisir des relations semblables à celle que l'on trouve en linguistique ou en physique : par exemple, à la suite de l'apparition du phonème $[\mathrm{m}]$, le trait distinctif « vocalique/non vocalique » peut uniquement entraîner 
la formation du phonème [1]; si on se donne toutes les variables d'une équation à l'exception d'une seule, alors cette dernière aura nécessairement une ou quelques valeurs déterminées - dans l'équation « $\mathrm{p}=\mathrm{m} \times \mathrm{g}$ », $\mathrm{si}$ « $\mathrm{m}=2$ » et « $\mathrm{g}=10$ », alors « $\mathrm{p}$ » ne peut être égal qu'à « 20 ». Il existe donc des structures dont le contenu des éléments dépend étroitement $\mathrm{du}$ contenu des autres éléments et des relations qu'ils entretiennent : si tous les éléments « $a, b$, c, etc. », d'une structure « $S$ » sont donnés à l'exception d'un seul, alors le contenu de ce dernier est entièrement déterminé par le contenu des autres éléments et des relations qu'ils entretiennent. On comprend que de telles structures aient conduit certains auteurs à nier la « subjectivité constituante » et à réduire le sujet humain (ou animal) à une " subjectivité constituée " ${ }^{17}$. Mais toutes les structures découvertes en sciences humaines et sociales ne s'apparentent pas à des structures phonologiques ou physiques. Ce n'est pas le cas de la structure du conditionnement. Même si on se donne un « Iimp » (obtenir des boulettes) et la relation de proximité spatio-temporelle, on ne peut savoir quelle position «I» le pigeon va adopter. Pour contraindre cette structure à ressembler à une structure phonologique ou physique, il faut contrôler artificiellement le nombre de «I » disponibles : le coup de sifflet dans l'expérience du chien et le levier dans celle du rat sont les seuls événements régulièrement présentés et susceptibles d'entrâner l'arrivée de la nourriture.

La structure du conditionnement est loin d'être la seule dans ce cas. Lorsque la relation ritualisée d'autorité entre l'oncle et le fils diminue au Moyen Âge, la relation ritualisée d'autorité entre le père et le fils augmente ${ }^{18}$. Souvenons-nous en effet qu'elles sont antithétiques. Le changement de statut de l'oncle semble, dès lors, déclencher pour ainsi dire automatiquement la modification du statut du père. Supposons maintenant que l'on connaisse le contenu du rite d'autorité entre l'oncle et le fils. Cela ne nous apprend rien sur le contenu du rite d'autorité entre le père et le fils. Ce dernier peut prendre des apparences diverses : la structure impose seulement à ce rite d'instituer entre le père et le fils une relation d'autorité. Patrice Maniglier soutient que la structure chez Lévi-Strauss et Saussure diffère profondément de la structure chez Jakobson, parce qu'elle possède nécessairement un caractère «bricolé » : elle n'existe pour ainsi dire qu'à travers les variations d'elle-même qu'elle ne cesse de produire ; elle est « toujours à refaire $»^{19}$.

Comme l'écrit Deleuze, il est en ce sens possible de "concilier genèse et structure ${ }^{20}$ ». Certaines structures imposent effectivement aux éléments certains rapports et par voie de conséquence certains contenus. Elles ne décident pas toutefois intégralement du contenu de chaque élément - le contenu des autres éléments étant donné. La norme sociale (l'ensemble des conduites autorisées au sein d'un groupe social) n'est pas en ce sens toujours préformée dans la structure. Certaines sociétés professent l'autochtonie des êtres humains ${ }^{21}$ (tout être humain naît de la terre), mais elles n'expriment évidemment pas cette croyance au moyen des mêmes personnages ou des mêmes histoires. Une structure en ethnologie n'existe qu'à travers l'une de ses variantes (tel rite, telle norme, etc.). Le chercheur ne rencontre jamais qu'une variante de cette structure et ne peut pas prédire le contenu d'un élément de cette variante, même s'il connaît tous les autres éléments et les relations qu'ils entretiennent. La structure n'est pas seulement, dans ce cas, une instance régulatrice imposant ou interdisant aux éléments tels ou tels contenus. Elle pose inconsciemment à l'individu ou au groupe social un problème de création: quelle apparence singulière allons-nous donner à cette relation que la structure nous impose? Quelle variante adopter? Quelle posture? Quel rituel? Quelle norme? Elle soumet le pigeon ou une société à une situation de 
recherche. Elle l'oblige à inventer une superstition, un rite, une norme, etc. Elle est donc moins de nature constituée que constituante. L'analyse structurale en ethnologie, comme en psychologie cognitive, ne nous invite donc pas à réduire le sujet à une marionnette achevée dont une structure inconsciente tirerait les ficelles. Certaines structures cognitives paraissent au contraire posséder «un caractère primitif et générateur ${ }^{22}$ » comme le remarque Piaget, à partir d'une relecture du structuralisme mathématique et de la psychologie de son temps. Bourdieu considère de la même manière, à partir de ses propres recherches en sociologie, que l'habitus est une " structure structurante » capable de générer des " pratiques et des représentations »"

Une structure ne soulève pas uniquement, en définitive, la question de son origine. Elle nous interroge aussi sur sa manifestation, c'est-à-dire sur la manière dont elle crée une variante d'elle-même. Certaines variantes sont prédites par la structure. Nous dirons que de telles structures sont "structurées". D'autres ne le sont guère, comme nous venons de le montrer ou comme le considèrent dans d'autres situations Saussure, Deleuze, Piaget et Bourdieu. La structure constitue, dans cette situation, les conditions d'un problème qu'un individu ou un groupe social doit résoudre. Nous qualifierons une structure de ce genre de «structurante " : la structure impose dans ce cas aux éléments certaines relations, mais ne leur impose pas totalement certains contenus. La question de la manifestation des variantes n'a pas été véritablement abordée en ethnologie. Notre examen de la structure du conditionnement suggère que des structures cognitives d'apprentissage structurantes pourraient être à l'origine de ces variantes. Aussi, une analyse à la fois structurale et cognitiviste pourrait permettre de comprendre de quelle manière les êtres humains génèrent des variantes d'une même structure sociale à partir de certains processus cognitifs comme la relation de proximité spatiotemporelle. Selon nous, c'est parce que le structuralisme classique s'est essentiellement intéressé, jusqu'à présent, aux structures structurées ou parce qu'il a délaissé la question de la formation des variantes que certains auteurs en sont venus à nier la "subjectivité constituante" et à réduire le sujet humain (ou animal) à une « subjectivité constituée $»^{24}$.

\subsection{Une nouvelle structure paradigmatique pour penser la genèse des structures en sciences humaines et sociales : les groupements configurés}

27 Comment expliquer la formation d'une structure ? Pourquoi un être vivant finit-il par imposer à certains éléments toujours les mêmes relations? Il ne suffirait pas toutefois de dévoiler la manière dont les structures sont élaborées pour résoudre le problème de leur genèse. On ne peut pas se contenter, en d'autres termes, au moyen d'une analyse structurale, de révéler les relations capables de produire des structures. Il faut aussi rendre compte, selon nous, du nombre relativement faible de structures engendrées par ces relations.

28 La structure du conditionnement chez les êtres humains peut associer n'importe quel « I » présent dans l'environnement à un « Iimp » et fabriquer ainsi potentiellement une infinité de superstitions. Autrement dit, une structure structurante possède de toute évidence une puissance créatrice importante, et on constate empiriquement qu'il existe effectivement un grand nombre de variations pour une même structure. En revanche, on observe très peu de structures. Il n'existe par exemple, selon Descola, que quatre 
grandes structures (animisme, totémisme, analogisme, naturalisme) régissant la façon dont un groupe social s'identifie et se différencie de ce qui n'est pas lui ${ }^{25}$. Pourquoi la créativité humaine est-elle si faible lorsqu'il s'agit d'inventer non plus des variantes d'une structure, mais des structures en tant que telles ? Voilà pourquoi il importe selon nous de soulever deux questions lorsqu'on pose le problème de la genèse des structures : quelles relations ont généré telle structure (nature des relations génétiques) et pourquoi n'en ont-elles constitué aucune autre (finitude des relations génétiques)? Autrement dit, on doit s'interroger simultanément sur la nature et sur la finitude des relations génératives de structures.

Les sciences humaines ne peuvent pas se donner pour tâche de comprendre la formation des structures biologiques ou physiques. Elles n'ont évidemment rien à dire sur la naissance des espèces vivantes ou des lois régissant les phénomènes matériels. C'est pourquoi les structures innées ne concernent pas les sciences humaines. Elles sont en effet le fruit de l'évolution des génomes. Elles ne résultent pas de structures d'apprentissage, à la différence des structures composées d'idées ou plus exactement de «représentations" conscientes ou inconscientes (normes sociales, théories scientifiques, mythes, etc.). Si elles souhaitent s'interroger sur l'origine des structures, les sciences humaines et sociales doivent donc se tourner vers des structures créées par les êtres humains. Comme nous n'en sommes encore qu'à un stade exploratoire, il pourrait être intéressant de rechercher des cas dans lesquels les relations génératives de structures seraient à la fois plus simples à identifier (problème de la nature de ces relations) et peu prolifiques (problème de la finitude de ces relations). Un exemple retient pour cette raison depuis plusieurs années notre attention: les groupements configurés (Figure 1). 
Figure 1. Une illustration de la notion de « configuration »

Dans la représentation sumérienne des quantités, le placement des symboles est " configuré » : il est possible d'anticiper la position du

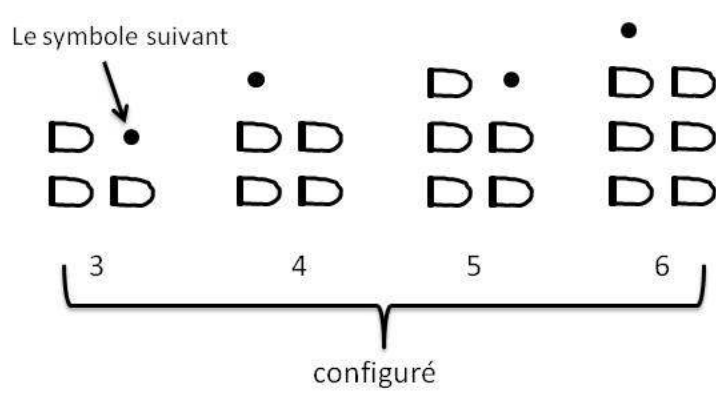
symbole suivant.

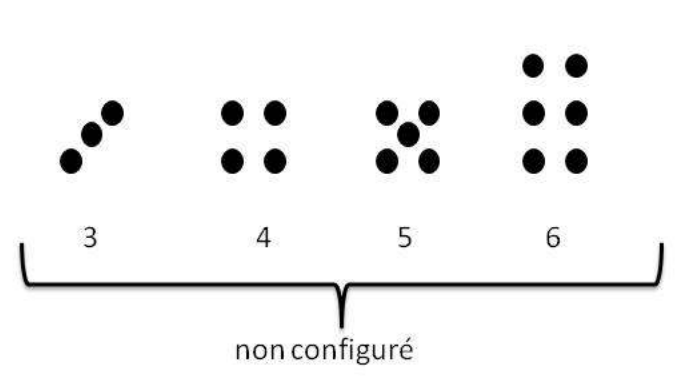

Sur un dé, le placement des symboles n'est pas configuré : il est impossible de deviner la place que va occuper le symbole suivant par la simple observation des positions des symboles antérieurs.

Adaptée de Miravete Sébastien, Tricot André, Kalyuga Slava \& Franck Amadieu, « Configured-group hypothesis: fast comparison of exact large quantities without counting », Cognitive Processing, International Quarterly of Cognitive Science, vol. 18, New York, Springer, 2017, p. 447-459

Les êtres humains possèdent, à l'instar de nombreuses espèces animales, un système numérique inné ${ }^{26}$. À l'aide de celui-ci, deux quantités d'objets peuvent approximativement être comparées. Il différencie par exemple très rapidement (250 millisecondes) 15 et 46 objets. Cependant, la différence entre 45 et 46 objets lui échappe toujours parce que les quantités sont trop proches. Un tel système prive les êtres humains de l'exactitude, et les chercheurs en cognition numérique essaient, entre autres, de comprendre de quelle façon les êtres humains parviennent à traiter avec précision des quantités d'objets plus importantes. Le recours au langage dans le domaine du dénombrement est le plus souvent mis en avant pour expliquer le passage de l'approximation à l'exactitude ${ }^{27}$. À la différence des autres espèces animales, les êtres humains mobilisent en effet des symboles numériques ( « un », « deux », « 34 », etc.) pour désigner des nombres d'objets. Cela leur donne en particulier le moyen de compter. Il est remarquable toutefois que le comptage dans certaines sociétés les limite à de très petites quantités: certains peuples de Nouvelle-Guinée emploient au maximum une trentaine de symboles numériques corporels (les doigts de la main, du pied, les coudes, etc. $)^{28}$. En outre, les données issues de l'archéologie et de la psychologie de l'éducation suggèrent que le traitement de grandes quantités d'objets ne se réduit pas à une simple procédure de comptage. Quelle que soit la zone géographique et quelle que soit l'époque, les êtres humains commencent en effet par organiser les grands nombres d'objets à l'aide de groupements configurés (Figure 2). Ils ne parviennent à l'exactitude qu'en adoptant, dans un premier temps, une certaine manière de se représenter les objets. Il ne suffit donc pas d'avoir des symboles numériques. Il importe aussi d'inventer une nouvelle façon de figurer efficacement les objets. Or, les relations entre 
les objets dans ces diverses représentations sont toujours les mêmes et suffisent à expliquer la raison pour laquelle ces représentations permettent de distinguer avec exactitude de grandes quantités d'objets ${ }^{29}$. Ces représentations sont donc homologues. Les groupements configurés ne sont rien d'autre que la manifestation d'une unique structure permettant de figurer avec exactitude de grands nombres d'objets. Comment cette structure idéelle a-t-elle été engendrée (nature des relations génératives) et pourquoi n'en existe-t-il qu'une seule dans toute l'histoire de l'humanité (finitude des relations génératives)?

Figure 2. Six exemples de groupements configurés issus des données de la psychologie de l'éducation (1a, 1b, 1c), de l'archéologie (1d, 1e) et de l'ethnologie expérimentale (1f)

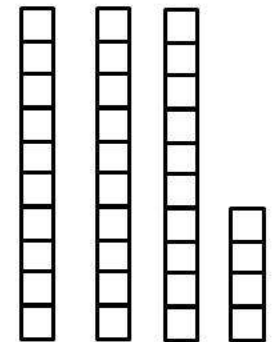

1a. La représentation du nombre 34 avec des barres de 10 (ten-blocks).

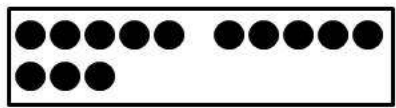

1b. La représentation du nombre 13 avec une grille de 20 (twenty-frame)

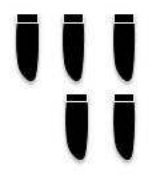

1e. La représentation du nombre 5 chez les Elamites

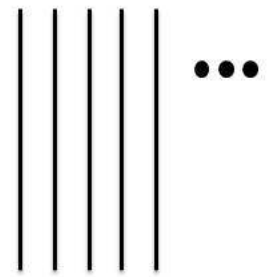

1c. La représentation du nombre 53 avec le modèle UDSSI

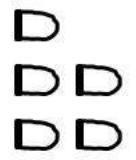

1d. La représentation du nombre 5 chez les Sumériens

Chacun de ces exemples est, selon notre hypothèse de recherche, une variante d'une même structure. Adaptée de Miravete Sébastien, Tricot André, Kalyuga Slava \& Franck Amadieu, « Configured-group hypothesis: fast comparison of exact large quantities without counting », Cognitive Processing, International Quarterly of Cognitive Science, vol. 18, New York, Springer, 2017, p. 447-459

Plusieurs solutions sont en effet envisageables et observables pour passer de l'approximation à l'exactitude. On peut par exemple aligner les objets les uns contre les autres $^{30}$ ou encore associer à un symbole un objet (entailles sur des os $^{31}$, comptage d'un en un, etc.). Ces procédures sont particulièrement coûteuses sur le plan matériel ou cognitif dès que l'on commence à dépasser une dizaine d'objets ${ }^{32}$. Notre hypothèse de recherche est que les groupements configurés (Figure 3) ont été obtenus par optimisation. Cette structure, que nous qualifierons de "restructurante», consiste à développer par tâtonnement de nouvelles structures de représentation en diminuant la charge cognitive (les ressources requises pour réaliser n'importe quel raisonnement) d'une part, et d'autre part en réduisant au minimum l'usage d'objets extérieurs au corps. Tout se passe comme si on découvrait de nouvelles manières de porter des charges plus lourdes en apprenant à positionner correctement notre corps par des essais successifs : on restreint l'effort musculaire au minimum et on évite de recourir à 
des outils (poulies, leviers, etc.). Notons que l'accroissement du rendement n'est pas pour autant dans ce cas le but poursuivi. Il n'est qu'un moyen pour développer de nouvelles capacités. Dans une telle approche, le tâtonnement permet d'explorer différentes formes de relations spatiales entre les objets. Mais il se soumet continûment aux limites de l'organisme lors de ses tentatives : il continue son exploration tant que l'appareil visuel demeure, dans notre exemple, incapable de différencier deux quantités très proches. Ces limites physiologiques et cognitives, propres à certaines aptitudes innées ou acquises, agissent alors comme des contraintes dont l'impact est de réduire drastiquement le nombre de solutions possibles. La contingence se soumet pour ainsi dire à la nécessité. C'est pourquoi, selon nous, les êtres humains ne pouvaient en dernier ressort développer qu'une seule manière de représenter les grands nombres d'objets - probablement la plus optimale au regard de leurs capacités perceptives et cognitives. À l'instar du rat de Skinner, ils n'ont sans doute pas eu d'autres options.

Figure 3. Exemple d'item proposé à des enfants de huit ans non entraînés

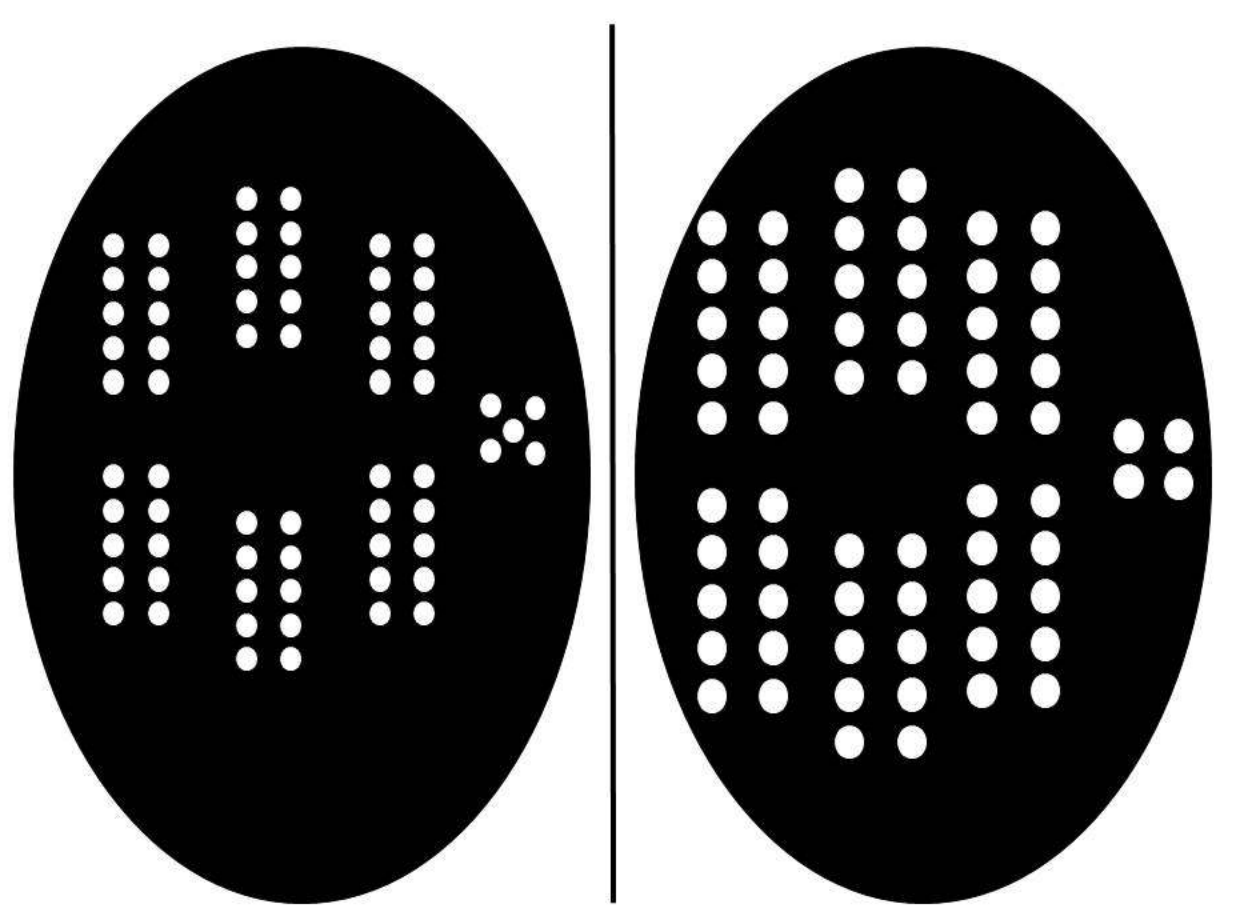

Les données montrent que les enfants parviennent aisément (plus de $90 \%$ de réussite) et en moins de 5 secondes (sans compter) à comparer 65 et 64 points, lorsque ces derniers sont organisés à l'aide groupements configurés, même lorsque le plus petit nombre de points occupe la surface la plus grande. Ces résultats suggèrent que les groupements configurés facilitent la représentation visuelle de deux grandes quantités très proches.

Adaptée de Miravete Sébastien, Tricot André, Kalyuga Slava \& Franck Amadieu, « Configured-group hypothesis: fast comparison of exact large quantities without counting », Cognitive Processing, International Quarterly of Cognitive Science, vol. 18, New York, Springer, 2017, p. 447-459

Une structure restructurante se définit par conséquent, dans ce cas précis, par une relation génétique entre une stratégie cognitive de création (le tâtonnement) et une ou plusieurs contraintes internes à l'organisme. Nous dirons par commodité qu'une structure restructurante se compose d'un système de production aléatoire de relations instables capables de façonner de nouveaux liens entre les éléments (le tâtonnement) et des contraintes internes (ou externes) à l'organisme dont le rôle est d'éliminer certains 
de ces liens (rôle régulateur) et de conserver les autres (rôle stabilisateur). C'est de cette façon, selon nous, qu'une nouvelle structure se forme par la cristallisation de liens apparus de manière en partie aléatoire: les contraintes limitent le nombre de liens produits hasardeusement par le tâtonnement d'une part, et d'autre part assurent la pérennité des liens les plus pertinents. C'est pourquoi, dans une structure restructurante, il s'avère impossible de penser séparément le processus de production de nouvelles relations et les contraintes servant à sélectionner et à préserver les relations les plus intéressantes. L'optimisation est une structure cognitive d'apprentissage restructurante, dans la mesure où elle cherche à imposer aux éléments certaines relations invariantes dans le but de satisfaire une contrainte précise - rendre possible, par exemple, la comparaison de deux grandes quantités d'objets.

Une structure structurante comme le conditionnement se contente, pour le dire brièvement, d'imposer aux éléments une relation invariante de proximité spatiotemporelle qu'elle n'a pas inventée - cette relation est sans doute mise en œuvre par une stratégie inconsciente et innée; cette dernière serait davantage le fruit de l'évolution des espèces que celui de l'histoire. Une structure restructurante génère la relation invariante qu'elle impose aux éléments. Elle engendre simultanément la structure au sens classique du terme et la variante de cette structure, c'est-à-dire la forme particulière que prend cette structure à travers l'emploi de certains objets ou de certains symboles (cf. Figure 2). Il est en effet impossible de tâtonner sans se donner des objets réels qui confèrent immédiatement une apparence particulière à la structure. Un groupe social finit toujours par préférer certains objets ou certains symboles à d'autres, pour figurer les groupements configurés. C'est le moment où il institutionnalise l'une des variantes que certains de ses membres ont créée.

L'étude des groupements configurés suggère, en dernière analyse, que les contraintes sont à l'origine du nombre relativement limité de structures produites par des relations instables telles qu'en engendre le tâtonnement. Devons-nous en déduire que seule une analyse structurale et cognitiviste est à même de résoudre le problème de la genèse et de la manifestation des structures?

\subsection{Revisiter les concepts de structure, d'interdisciplinarité et d'écologie}

\subsubsection{Trois types de structure}

Ces travaux sur le conditionnement et les groupements configurés nous invitent, en somme, à supposer l'existence de trois genres de structures : les structures structurées, les structures structurantes, les structures restructurantes. Dans le premier cas, les relations et les contraintes se confondent pour ainsi dire. Les relations entre les éléments sont, en d'autres termes, si contraignantes qu'il devient possible de prédire le contenu des éléments : si tous les éléments « a, b, c, etc.» sont donnés à l'exception d'un seul, alors le contenu de ce dernier est entièrement déterminé par le contenu des autres éléments et des relations qu'ils entretiennent. Nous dirons que de telles relations invariables sont «stables » (incapables de produire de nouvelles structures) et "contraignantes» (incapables de produire des variantes imprévisibles) et qu'elles correspondent aux relations que l'on trouve généralement dans les lois de la physique et en phonologie. 

sont pas suffisamment contraignantes en tant que telles : il n'est pas possible de prédire le contenu des éléments, même si l'on se donne l'ensemble des éléments à l'exception d'un seul. Certaines contraintes (géographiques, physiologiques, cognitives, etc.) limitent toutefois le choix des éléments. Il est même possible de restreindre ce choix à quelques éléments dont on peut prévoir le contenu. La structure structurante se comporte alors comme une structure structurée, sans pour autant devenir une structure structurée : les relations stables ne deviennent pas contraignantes; elles sont seulement extrêmement contraintes. Nous dirons in fine que de telles structures, semblables à la structure du conditionnement, se composent d'une "relation génétique " entre des relations «stables» (incapables de produire de nouvelles structures) «non contraignantes" (capables de produire des variantes imprévisibles) et des « contraintes » internes ou externes à l'organisme plus ou moins importantes.

Dans le troisième cas, on trouve tout d'abord au sein de la structure des relations à la fois «instables» (capables de produire de nouvelles structures) et «non contraignantes » (capables de produire des structures ou des variantes imprévisibles). Ces relations sont ensuite soumises à des contraintes internes ou externes à l'organisme. Ces dernières opèrent une sélection au niveau des éléments, mais aussi au niveau des relations instables. C'est pourquoi la relation génétique entre les relations instables et les contraintes finit par engendrer une structure structurante (certaines relations parmi l'ensemble des relations instables sont sélectionnées et conservées) et sa variante (certains éléments parmi l'ensemble des éléments disponibles sont sélectionnés et conservés). Une structure restructurante se constitue donc, en définitive, d'une relation génétique entre des relations instables non contraignantes et des contraintes, mais aussi de relations stables et non contraignantes (la structure structurante) qui ne sont rien d'autre que le fruit de cette relation génétique. Ces dernières peuvent parvenir à se détacher de la structure restructurante par l'exercice de certaines contraintes (imposant au système cognitif de conserver ses créations et de les réemployer) et former ainsi une structure structurante indépendante de son lieu d'élaboration.

\subsubsection{Contraintes structurales et interdisciplinarité}

Les contraintes sont par conséquent des composantes essentielles des structures structurantes et restructurantes - lorsqu'elles ne se confondent pas purement et simplement avec les relations invariantes dans le cas d'une structure structurée. Nous n'avons pas encore toutefois précisé leur contenu. Nous allons voir en outre que l'existence et la nature même de ces contraintes au cœur de chaque structure nous conduit à repenser les notions d'interdisciplinarité et d'écologie. Les contraintes présentes par exemple dans la structure structurante du conditionnement imposent aux «I» (les postures du pigeon) d'être proches dans le temps et dans l'espace d'un «Iimp » (l'arrivée de nourriture), d'être au moins prédictifs (la posture doit favoriser dans la plupart des cas l'arrivée de nourriture) et d'exister (cette contrainte oblige le pigeon à choisir un «I I si aucune contrainte externe ne limite ses choix) ; elle n'impose pas toutefois au «I» tel ou tel contenu (dans une situation réelle, les «I I disponibles sont multiples). Chez des êtres humains, le «I " peut par exemple avoir été inventé ou sélectionné par un ou plusieurs individus. Il est ensuite transmis aux autres membres du groupe au moyen d'une technique de contrôle quelconque consistant à réduire le 
nombre de «I" possibles à un seul (cf. note 16). C'est de cette façon que la nouvelle superstition (tel «I» entraîne tel «Iimp») se propage ou du moins pourrait se propager.

Dans une telle situation, le choix du «I » et de sa diffusion est soumis à un ensemble de contraintes internes ou externes: la présence de ce "I» dans l'environnement (contrainte externe); la confiance que l'on accorde à telle personne qui nous présente ce «I» comme le bon (contrainte interne); la constatation plus ou moins objective que ce «I» produit le «Iimp» (contrainte externe), etc. Toutes ces contraintes s'apparentent à des relations invariantes et relativement stables dans la mesure où elles peuvent imposer, comme nous venons de l'expliquer, la sélection de certaines relations ou de certains éléments. Elles pourraient donc faire l'objet à leur tour d'une analyse structurale si on suppose qu'elles ne sont rien d'autre que des structures structurantes ou structurées. Or, de telles contraintes, que nous pouvons qualifier dès lors de "structurales", n'appartiennent pas nécessairement au champ des sciences cognitives: elles peuvent être de nature physique, biologique, géographique, géologique, sociologique, historique, etc. Aussi, une structure structurante, comme celle du conditionnement, est-elle impensable indépendamment des relations qu'elle entretient intérieurement avec d'autres structures (les contraintes étant des composantes de la structure) et qui participent à sa manifestation, c'est-à-dire à la formation de sa variante.

De la même manière, on ne peut pas séparer la structure restructurante des contraintes structurales d'origine diverses qui lui permettent d'engendrer une structure structurante. Les sciences cognitives peuvent certes mettre au jour les structures cognitives d'apprentissage susceptibles de créer des structures idéelles ou leurs variantes. Elles ne peuvent en revanche rendre compte de l'existence et de la structure de toutes les contraintes composant ces structures d'apprentissage. Ses méthodes ne lui permettent pas de savoir, par exemple, pour quelle raison les êtres humains ont eu besoin de traiter avec exactitude de grandes quantités de ressources. Seules les données historiques suggèrent que ce traitement apparaît vers la fin du $\mathrm{IV}^{\mathrm{e}}$ siècle avant notre ère, avec la formation des premiers royaumes ${ }^{33}$, c'est-à-dire avec l'apparition d'organisations sociales nécessitant sans doute une comptabilité à plus grande échelle. Cette contrainte explique pourtant pour quelle raison on cherche par le tâtonnement à produire une représentation exacte des grandes quantités.

41 C'est pourquoi, un tel cadre de recherche (soucieux de rendre compte, au moyen d'une analyse structurale, de la genèse des structures et de leurs variantes) en sciences humaines et sociales ne peut être qu'interdisciplinaire: les structures susceptibles d'exercer une contrainte sur une autre n'appartenant jamais qu'à un seul domaine de recherche. Une discipline peut donc se développer indépendamment (découvrir des relations invariantes stables, commencer à mettre au jour certaines relations instables), jusqu'à rencontrer une contrainte inexplicable par ses propres méthodes ou objets d'étude. Le recours à d'autres disciplines se révèle alors indispensable et même relativement fécond: nous avons essayé de l'illustrer en mobilisant en sciences cognitives les données issues de l'archéologie, de l'ethnologie, de l'histoire et de la psychologie de l'éducation pour comprendre la genèse des groupements configurés. Les sciences cognitives semblent alors apporter en retour un certain éclairage sur les processus cognitifs d'apprentissage (les relations non contraignantes comme la relation de proximité spatio-temporelle ou le tâtonnement) susceptibles de produire, par 
exemple, de nouvelles variantes idéelles (diverses superstitions) ou de restructurer des représentations sociales (la manière dont un groupe social se représente avec exactitude de grandes quantités d'objets).

L'interdisciplinarité devient ainsi "transindividuelle» au sens défini par Simondon ${ }^{34}$. Les disciplines partagent des informations vitales pour leur développement. Autrement dit, elles se transmettent des "germes » (les structures issues d'autres disciplines) capables d'ensemencer leurs "métastabilités» (les contraintes ou les relations non contraignantes dont elles ne peuvent rendre compte). Elles intègrent localement ${ }^{35} \mathrm{de}$ nouvelles perspectives et se restructurent alors en partie à l'aide d'autres disciplines. Elles « communiquent » ou se " co-individuent » de cette façon, sans devenir une seule et même discipline. La dépendance des structures à l'égard d'autres structures (contraignant ou générant ces structures) oblige en définitive les disciplines à s'ouvrir dynamiquement aux autres. Cette ouverture ne revient pas à les soumettre à un métarécit anthropologique susceptible de les réduire à une méthodologie ou à une explication commune. Il ne s'agit en aucun cas de les intégrer dans une théorie ou une méthodologie unique. Les disciplines conservent leurs spécificités irréductibles sans pour autant se fermer aux autres, et ne continuent même à se développer qu'au contact des autres (interdisciplinarité transindividuelle et locale).

Autrement dit, une anthropologie cherche moins, dans une telle approche, à uniformiser les sciences humaines et sociales au moyen d'un paradigme commun qu'à les connecter autour de certaines problématiques communes - la compréhension de tel ou tel phénomène social, de tel ou tel comportement humain, etc. De plus, elle ne cantonne pas a priori son champ d'investigation aux seules sciences humaines et sociales. Elle n'opère en ce sens aucune séparation entre les sciences traditionnelles de la nature (physique, biologie) et celles de la culture (histoire, sociologie, psychologie, etc.) - la physique et la biologie lui offrent par exemple l'opportunité de comprendre certaines contraintes qui s'exercent sur l'être humain et qui participent pour cette raison à l'évolution de ses idées, de ses représentations sociales, de ses habitudes, etc.

\subsubsection{Relations génétiques et écologie}

Enfin, les êtres humains sont loin d'être la seule espèce à être capable de produire des objets culturels. Comme nous l'avons vu, les pigeons peuvent développer des superstitions. Lévi-Strauss reconnaît le caractère artificiel de l'opposition entre nature et culture, après avoir insisté dans un premier temps sur celle-ci ${ }^{36}$.

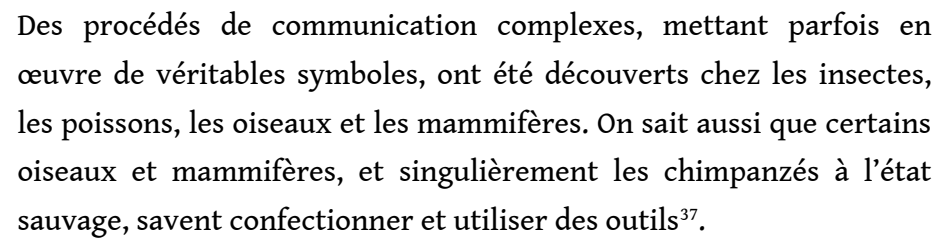

Nous pourrions donc chercher à étendre le concept de structure structurante, ou même celui de structure restructurante. Ce dernier pourrait probablement être repris par les sciences physiques et technologiques par exemple. Cette perspective n'est pas sans rappeler la description que propose simondon du processus d'individuation d'une brique: l'argile travaillée par l'artisan (relations instables) entre par exemple en contact avec un moule (contrainte); le moule opère en retour une contrainte sur 
l'argile et sa forme se propage ainsi au sein d'une masse argileuse homogène susceptible de l'accueillir (relation génétique) ${ }^{38}$. Certains chercheurs en biologie et en mathématiques ont récemment proposé de repenser le fonctionnement d'un être vivant dans une perspective relativement proche de la nôtre : ils ne perçoivent plus les organismes comme des structures structurées semblables à une structure physique (cf. 2.1.) mais plutôt comme des structures restructurantes ${ }^{39}$.

Il serait donc possible de repenser de cette manière les structures mises au jour par les sciences de la matière et du vivant. Une telle perspective reviendrait en dernière instance à revisiter l'écologie, c'est-à-dire les relations intra et inter systémiques dont un écosystème (biocénose et biotope) est constitué. Un écosystème se compose en effet, dans une telle approche, de structures structurées, structurantes et restructurantes en relation - les structures structurantes et restructurantes étant les seules à posséder une puissance génétique susceptible de créer des variations ou des structures imprévisibles. Il n'est donc pas une unique structure structurée, structurante ou même restructurante, mais un réseau de structures qui participent (par leur puissance génétique ou en exerçant des contraintes) à la création continue de variations et de structures, et ainsi au développement de cet écosystème. Dès lors, l'écologie n'est pas seulement, dans un tel cadre, la science des « interactions » (cf. 1.3.) entre des systèmes noués eux-mêmes d'interactions (écologie interactionnaliste) - mais plutôt celle des structures. Elle n'est pas, pour cette raison, uniquement relationnaliste ou holistique, comme le propose pourtant à juste titre « l'écologie profonde ${ }^{40}$ ». Elle n'est pas non plus la science visant à réduire les relations intra et inter systémiques à des structures structurées (écologie structuraliste classique). Elle est plus précisément la science des relations génétiques qu'entretiennent des relations stables ou instables (mais non contraignantes) et des contraintes structurales spécifiques, c'est-à-dire la science de ces relations génétiques connectées entre elles qui créent et régissent les différentes structures et les multiples variations dont se tisse un écosystème (écologie des relations génétiques).

\subsubsection{Science et philosophie}

47 Cette science des relations génétiques n'est pas sans rappeler certaines thèses esquissées par Simondon. Comme le résume Jean-Hugues Barthélémy :

Le projet d'une allagmatique, déjà formulé par Simondon dans les passages de ses thèses où il dialogue avec la cybernétique, rapproche donc son projet philosophique de l'idée d'une science [...] Tandis que chaque science positive est une science de structures génériques, l'allagmatique est la science des opérations génétiques ${ }^{41}$.

Notre hypothèse est plutôt que "chaque science positive " peut devenir, dans une certaine mesure, une science des "opérations génétiques" (des structures structurantes ou restructurantes). Comme nous venons de l'illustrer (cf. 2.1. et 2.2.) dans le cadre d'une analyse cognitiviste et structuraliste de certains phénomènes sociaux, une discipline scientifique peut dévoiler des relations stables non contraignantes (la relation de proximité spatio-temporelle) ou instables (le tâtonnement) susceptibles d'engendrer respectivement des variantes inédites d'une structure (différentes superstitions chez le pigeon) ou de nouvelles structures (les groupements configurés). Chaque science positive ne se réduit donc pas à l'étude des "structures génériques " (des structures structurées) et peut même s'ouvrir localement 
(cf. 2.3.2.) à d'autres disciplines. Elle ne se définit alors plus momentanément «par un domaine objectif comme étude de la matière, étude de la vie... ${ }^{42}$ ».

Il serait en outre sans doute possible aujourd'hui de modéliser formellement de telles relations génétiques : ainsi, certains mathématiciens travaillent déjà sur l'émergence de structures sociales ${ }^{43}$. Une telle approche exclusivement scientifique présente néanmoins une limite selon nous : ces modélisations mathématiques pourraient encore faire l'objet d'une critique d'inspiration bergsonienne rappelant leur caractère intempore ${ }^{44}$. Il y a plus généralement quelque chose qui échappe à l'analyse purement scientifique (mais non nécessairement à toute analyse structurale) dans n'importe quel processus de création. La philosophie demeure encore, pour cette raison, une " méthode ${ }^{45}$ » de réflexion indispensable lorsqu'on souhaite penser, entre autres, la temporalité ou la nature même des processus de création. Une anthropologie des relations génétiques, ou plus globalement une science des relations génétiques, ne peut donc se construire indépendamment de la philosophie ${ }^{46}$.

\section{Conclusion}

50 Le structuralisme classique en sciences humaines et sociales (cf. 1) s'est en résumé désintéressé de la créativité des structures (cf. 2.1.) et de leur genèse (cf. 2.2.) et a ainsi parfois donné l'impression de réduire la subjectivité humaine à une «subjectivité constituée » et le sujet humain à un " pur effet ». C'est pourquoi le post-structuralisme a sans doute été chez de multiples auteurs une tentative de réintroduire la "subjectivité constituante». Certains ont alors cherché à élaborer une subjectivité humaine en partie irréductible à toute forme de structure ${ }^{47}$ - cette partie irréductible pouvant servir à modifier ou à engendrer des structures. D'autres ont essayé de repenser le concept de structure et de lui restituer sa dimension génétique ${ }^{48}$. Chez ces derniers, le post-structuralisme est un (post-)structuralisme dans la mesure où il s'oppose moins au structuralisme dans son ensemble qu'au structuralisme classique. Nous espérons avoir contribué à son élaboration en y apportant quelques éléments de réflexion issus de nos recherches philosophiques et scientifiques en linguistique, en ethnologie et en sciences cognitives et sociales.

\section{BIBLIOGRAPHY}

Balibar Étienne, «Le structuralisme : une destitution du sujet ? ", Revue de métaphysique et de morale, $\mathrm{n}^{\circ}$ 45, Paris, Puf, 2005.

Barthélémy Jean-Hugues, Simondon, Paris, Les Belles Lettres, 2014.

- «Glossaire Simondon : les 50 grandes entrées dans l'œuvre », Appareil [En ligne], 16 | 2015, mis en ligne le 9 février 2016, consulté le 5 février 2019. URL : http://journals.openedition.org/ appareil/2253, DOI : 10.4000/appareil.2253.

Bourbaki Nicolas, Éléments d'histoire des mathématiques, New York, Springer, 2007. 
Bourdieu Pierre, Le Sens pratique, Paris, Éditions de Minuit, 1980.

Dehaene Stanislas, La Bosse des maths, Paris, Odile Jacob, 2010.

Deleuze Gilles, Différence et répétition, Paris, Puf, 2005.

Descola Philippe, « Entrevue ». Gruppen, n 7, Paris, Gruppen éditions, 2013.

- Par-delà nature et culture, Paris, Gallimard, 2015.

Frank Michael C., Fedorenko Evelina, Lai Peter, Saxe Rebecca \& Edward Gibson, « Verbal interference suppresses exact numerical representation », Cognitive Psychology [En ligne], vol. 64, Issue 1-2, p. 74-92, mis en ligne en 2012, consulté le 05 février 2019, https://doi.org/10.1016/ j.cogpsych.2011.10.004.

Hyde Daniel C., « Two systems of non-symbolic numerical cognition », Frontiers in Human Neuroscience [En ligne], vol. 5, mis en ligne le 29 novembre 2011, consulté le 4 février 2019, https://doi.org/10.3389/fnhum.2011.00150.

Ifrah Georges, Histoire universelle des chiffres. Tome I. Paris, Robert Laffont, 1994.

Izard Véronique \& Stanislas Dehaene, «Calibrating the mental number line », Cognition [En ligne], vol. 106, Issue 3, mis en ligne en 2008, consulté le 4 février 2019, https://doi.org/10.1016/ j.cognition.2007.06.004.

Jakobson Roman, Essais de linguistique générale. Les fondations du langage, Paris, Les Éditions de Minuit, 2003.

Lévi-Strauss Claude, Anthropologie structurale, Paris, Plon, 1974.

— Les Structures élémentaires de la parenté, Paris, EHESS, 2002.

— Le Totémisme aujourd'hui, Paris, Puf, 2011.

Lévi-Strauss Claude \& Didier Eribon, De près et de loin, Paris, Odile Jacob, 2009.

Maniglier Patrice, La Vie énigmatique des signes, Paris, Léo Sheer, 2006.

Manzo Gianluca, « Actions, interactions et structure dans l'émergence de la stratification sociale des diplômes : un modèle de choix discrets avec externalités ", Mathématiques et sciences humaines [En ligne], 175 | Automne 2006, mis en ligne le 11 décembre 2006, consulté le 5 février 2019. URL : http://journals.openedition.org/msh/3549

Miravete Sébastien, « La durée bergsonienne comme nombre spécial » dans Worms Frédéric (dir.), Annales bergsoniennes V, Paris, Puf, 2012.

- «Le postbergsonisme de Deleuze et Lyotard », dans Enaudeau C. \& F. Fruteau De Laclos, Différence, différend: Deleuze et Lyotard. Paris, Encore marine, 2015

— «Tetsugaku to Ninchi-shinrigaku wo Teigi suru: Berukuson Tetsugaku no Chikaku Riron to Burūnā no Ninchi-shinrigaku no Bunseki wo Tōshite » [« Définir la philosophie et la psychologie cognitive par l'analyse des théories de la perception issues de la philosophie de Bergson et de la psychologie cognitive de Bruner »], dans Hirai Y., Fujita H. \& S. Abiko (eds.), Shusuke Yamane. Berukuson Busshitsu to Kioku wo Kaibou suru: Gendai Chikaku Riron, Jikan-ron, Kokoro no Tetsugaku tono Setsuzoku [L'anatomie de Matière et mémoire de Bergson : connexions avec les théories contemporaines de la perception, de l'esprit et du temps], Tokyo, Soshi Shinsui, 2016. Texte traduit en japonais par Shusuke Yamane.

- «Bergson, Jankélévitch : une opposition sur le concept de temps » dans Bastiani F. (dir.)

Bergson, Jankélévitch et Levinas, Paris, Éditions Manucius, 2017. 
Miravete Sébastien, André Tricot, Kalyuga Slava \& Franck Amadieu, « Configured-group hypothesis: fast comparison of exact large quantities without counting », Cognitive Processing, International Quarterly of Cognitive Science, vol. 18, New York, Springer, 2017.

Montévil Maël, Longo Giuseppe \& Ana Soto, « Du siècle du gène à celui de l'organisme : introduction à de nouvelles perspectives théoriques ", dans Gaudin Thierry, Lacroix Dominique, Maurel Marie-Christine \& Jean-Charles Pomerol (dir.), Sciences de la vie, sciences de l'information (Colloque de Cerisy), Paris, ISTE-Éditions, 2017.

Montebello Pierre, Métaphysiques cosmomorphes - La fin du monde humain, Dijon, Les presses du réel, 2015.

Naess Arne, "The shallow and the deep, long-range ecology movement. A summary ", Inquiry, [en ligne], vol. 16, Issue 1, p. 95-100, mis en ligne le 29 aout 2008, consulté le 5 février 2019, https://doi.org/10.1080/00201747308601682.

Piaget Jean, «Les structures mathématiques et les structures opératoires de l'intelligence », dans Piaget jean, Beth Evert W., Dieudonné Jean, Lichnerowicz André, Choquet Gustave \& Caleb Gattegno (dir.). L'Enseignement des mathématiques. Nouvelles perspectives, Paris, Delachaux et Niestlé, 1955.

Rabouin David, «Structuralisme et comparatisme en sciences humaines et en mathématiques : un malentendu? », dans Maniglier Patrice (dir.), Le Moment philosophique des années 1960 en France, Paris, Puf, 2011.

Reznikova Zhanna \& Boris Ryabko, « Numerical competence in animals, with an insight from ants ", Behaviour [En ligne], 148(4), p. 408-434, mis en ligne en 2011, consulté le 14 janvier 2018, DOI:10.1163/000579511X568562.

Saussure Ferdinand de, Cours de linguistique générale. Paris, Payot et Rivages, 1995.

Simondon Gilbert, L'Individuation à la lumière des notions de forme et d'information. Grenoble, Éditions Millon, 2005.

Skinner Burrhus Frederic, " "Superstition" in the pigeon », Journal of Experimental Psychology, $\mathrm{n}^{\circ}$ 38, Washington, APA, 1948

Troubetzkoy Nicolas, « La phonologie actuelle », Journal de Psychologie Normale et Pathologique, $\mathrm{n}^{\circ} 30,1933$

\section{NOTES}

1. Lévi-Strauss n'est pas le premier à faire de la linguistique une science sociale (Anthropologie structurale, Paris, Plon, 1974, p. 46). Saussure considérait que la linguistique n'était qu'une des composantes de cette «science qui étudie la vie des signes au sein de la vie sociale [...]». Cette science ne serait même à ses yeux qu'une " partie de la psychologie sociale » (Cours de linguistique générale, Paris, Payot et Rivages, 1995, p. 33).

2. «La conviction des néogrammairiens était bien que le phonème, l'élément articulatoire inférieur et à la phrase et au mot, fournissait une base empirique à la linguistique. Le réel du langage serait alors physiologique dans ce sens-là. Mais Saussure établit, à travers une critique de la pratique "phonologique", l'impossibilité de trouver, sur la base de la seule observation de l'acte articulatoire, des "unités immédiatement données" élémentaires. C'est donc par cette "critique de la raison phonétique" que Saussure attaque le cœur du projet néogrammairien.» Maniglier Patrice, La Vie énigmatique des signes, Paris, Léo Sheer, 2006, p. 99-100. 
3. Nous sommes loin d'être les premiers à souligner le caractère structural de la méthode saussurienne. Ibid., p. 39-41.

4. Du point de vue de Jakobson, ce sont les traits distinctifs, et non les simples transitions d'un son à un autre, qui produisent les changements de signification. À titre illustratif, voir l'exemple qu'il donne du dialogue entre Alice et le Chat: «Vous avez dit cochon ou cocon?» (Essais de linguistique générale. Les fondations du langage, Paris, Les Éditions de Minuit, 2003, p. 46).

5. Ibid., p. 128-130.

6. Lévi-Strauss confie dans un entretien que la méthode structurale lui a été inspirée par la lecture de l'ouvrage Forme et croissance (1917) du mathématicien et biologiste D'Arcy Thompson (Lévi-Strauss Claude \& Eribon Didier, De près et de loin, Paris, Odile Jacob, 2009, p. 158-159). Ce dernier recourt à la notion géométrique de transformation pour identifier des identités de rapport entre les espèces, les organes, etc. - une transformation consiste à transformer une figure en une autre tout en conservant certains rapports entre les points. Lévi-Strauss s'inspire manifestement d'auteurs qui mobilisent les mathématiques pour mettre au jour des identités de rapport au sein de la matière organique (D'Arcy Thompson). Ajoutons que Lévi-Strauss rappelle systématiquement l'importance méthodologique des notions synonymes pour lui de "structure", de "forme" ou encore de «relation»: à titre d'exemple, voir Le Totémisme aujourd'hui, Paris, Puf, 2011, p. 18 et Les Structures élémentaires de la parenté, Paris, EHESS, 2002, p. 148.

7. Lévi-Strauss recourt très souvent à cet exemple résumant certains travaux de Radcliffe-Brown pour décrire la notion de structure en ethnologie - nous l'utilisons à notre tour afin de ne pas alourdir notre exposé. Les structures de la parenté sont évidemment beaucoup plus complexes (cf. Lévi-Strauss Claude, Anthropologie structurale, Paris, Plon, 1974, p.56-62), même si elles décrivent toutes des relations antithétiques saisies au moyen d'une analyse structurale.

8. Ibid., p. 54

9. Il serait absurde, aux yeux du structuralisme, de faire du récit minutieux des interactions une activité vaine. Cette dernière est au contraire pour lui sa condition de possibilité méthodologique et épistémologique : son lieu d'élaboration et de vérification. C'est seulement de cette manière que l'analyse structurale ne devient pas une procédure abusive de simplification: il ne faut jamais qu'une relation invariante dissimule des systèmes auxquels elle ne s'applique pas ; c'est pourquoi l'analyse structurale doit commencer par se donner un grand nombre de systèmes dont les interactions entre leurs éléments ont été suffisamment et correctement décrites.

10. Descola Philippe, « Entrevue », Gruppen $n^{\circ} 7$, Paris, Gruppen éditions, 2013.

11. Cela ne concerne sans doute pas uniquement les sciences empiriques (physique, biologie, sciences humaines). Les mathématiques semblent avoir adopté progressivement, depuis le $\mathrm{XIX}^{\mathrm{e}}$ siècle, une approche similaire (Bourbaki, Éléments d'histoire des mathématiques, New York, Springer, 2007, p. 36) et en avoir pris pleinement conscience en France grâce aux travaux de Bourbaki. Les structures mathématiques désignent en effet des relations invariantes entre les éléments de plusieurs problèmes. Celles-ci permettent surtout de résoudre plus simplement différents problèmes. C'est pourquoi, la différence entre le structuralisme empirique et mathématique paraît moins tranchée lorsque le structuralisme dans son ensemble est assimilé à un «instrument d'exploration», comme le soutient David Rabouin («Structuralisme et comparatisme en sciences humaines et en mathématiques : un malentendu? ", dans Maniglier Patrice (dir.), Le Moment philosophique des années 1960 en France, Paris, Puf, 2011, p. 13).

12. Il ne servirait à rien de conserver un langage ordinaire pour décrire non formellement une structure. Ce n'est pas parce qu'on écrit «si le père a une relation d'autorité avec le fils alors l'oncle doit avoir une relation de tendresse » à la place de «si p a f alors o $t \mathrm{f}$ » qu'on évite de cette façon d'énoncer une loi logique. Les symboles ne servent qu'à simplifier la formulation des relations formelles d'opposition que perçoit l'ethnologue.

13. Nicolas Troubetzkoy, « La phonologie actuelle », Journal de Psychologie, n 30, 1933. 
14. Dans une approche cognitiviste, un stimulus ne déclenche en aucun cas mécaniquement une représentation ou une réponse. C'est le système cognitif qui interprète en permanence les stimuli qu'il reçoit à l'aune des connaissances innées ou acquises dont il dispose - on parle alors de «traitement de l'information». Le stimulus est toujours l'élément d'un processus actif de décision. Il demeure à ce titre une "information ", c'est-à-dire une source de connaissance à partir de laquelle le système cognitif choisit de faire telle ou telle chose en la comparant à d'autres.

15. Skinner Burrhus Frederic, " "Superstition" in the pigeon ", Journal of Experimental Psychology, $\mathrm{n}^{\circ}$ 38, Washington, APA, 1948.

16. Une publicité ou une propagande efficace revient toujours à co-présenter de manière régulière une information " $\mathrm{I}$ » et une information "Iimp» (dans une affiche, une vidéo, un discours, etc.) positive (source de plaisir) ou négative (source de déplaisir ou de souffrance) présente dans les besoins ou la culture d'un groupe social. L'information « I » est alors l'objet que l'on cherche à faire consommer. Elle peut être aussi le groupe social ou l'attitude que l'on souhaite valoriser (au moyen d'une «Iimp» positive) ou rejeter (au moyen d'une "Iimp » négative). Les stéréotypes et adages («I implique Iimp») s'acquièrent selon nous de la même manière, par l'intermédiaire généralement d'une autorité (des proches ou une institution représentant consciemment ou inconsciemment aux yeux de l'individu une source d'information «I » pertinentes) qui les colporte de façon régulière à l'individu (consciemment ou non).

17. Balibar Étienne, «Le structuralisme : une destitution du sujet?», Revue de métaphysique et de morale, $\mathrm{n}^{\circ}$ 45, Paris, Puf, 2005, p. 15.

18. Lévi-Strauss Claude, Anthropologie structurale, Paris, Plon, 1974, p. 56

19. Maniglier Patrice, La Vie énigmatique des signes, Paris, Léo Sheer, 2006, p. 441.

20. «Il suffit de comprendre que la genèse ne va pas d'un terme actuel, si petit soit-il, à un autre terme actuel dans le temps, mais du virtuel à son actualisation, c'est-à-dire de la structure à son incarnation, des conditions de problèmes aux cas de solution [...]». Deleuze, Différence et répétition, Paris, Puf, 2005, p.237-238. Il est remarquable que Deleuze assimile la structure, comme nous souhaitons le faire, aux « conditions de problèmes » et non à une sorte d'instance de régulation, dont le rôle serait simplement d'interdire certaines pratiques ou de prescrire certaines attitudes, représentations ou émotions. Deleuze pose, de notre point de vue, dans ce passage le problème tout aussi fondamental de la manifestation de la structure, plutôt que celui de la genèse des structures. Il confère en outre à la structure un rôle créateur et non uniquement régulateur dans la mesure où elle tend déjà à "s'incarner" dans une forme (norme sociale, représentation, etc.) dont elle ne peut prévoir le contenu. C'est pourquoi la création de cette forme est pour elle un problème : la variante n'est pas préformée dans la structure ; la structure doit créer sa variante.

21. Lévi-Strauss Claude, Anthropologie structurale, Paris, Plon, 1974, p. 246-247.

22. « [...] il ne faut pas oublier que, psychologiquement, l'ordre de la prise de conscience renverse celui de la genèse : ce qui est premier dans l'ordre de la construction apparaît en dernier à l'analyse réflexive, parce que le sujet prend conscience des résultats de la construction mentale avant d'en atteindre les mécanismes intimes. Loin de constituer un argument décisif en faveur de l'indépendance des "structures" par rapport au travail de l'intelligence, leur découverte tardive et quasi inductive tendrait donc au contraire à faire soupçonner leur caractère primitif et générateur.", Piaget Jean, «Les structures mathématiques et les structures opératoires de l'intelligence ", dans Piaget jean, Beth Evert W., Dieudonné Jean, Lichnerowicz André, Choquet Gustave \& Caleb Gattegno (dir.). L'Enseignement des mathématiques. Nouvelles perspectives, Paris, Delachaux et Niestlé, 1955, p. 14.

23. "Les conditionnements associés à une classe particulière de conditions d'existence produisent des habitus, systèmes de dispositions durables et transposables, structures structurées prédisposées à fonctionner comme structures structurantes, c'est-à-dire en tant que 
principes générateurs et organisateurs de pratiques et de représentations qui peuvent être objectivement adaptées à leur but sans supposer la visée consciente de fins et la maîtrise expresse des opérations nécessaires pour les atteindre, objectivement réglées et régulières sans être en rien le produit de l'obéissance à des règles, et, étant tout cela, collectivement orchestrées sans être le produit de l'action organisatrice d'un chef d'orchestre. ", Bourdieu Pierre, Le Sens pratique, Paris, Éditions de Minuit, 1980, p. 88.

24. Balibar Étienne, «Le structuralisme : une destitution du sujet?", Revue de métaphysique et de morale, $\mathrm{n}^{\circ}$ 45, Paris, Puf, 2005, p. 15.

25. Dans le totémisme, autrui (humain ou non humain) possède en résumé une intériorité (idées, rêves, principes immatériels, affects, pensées, etc.) et une physicalité (processus physiologiques, apparences extérieures, principes matériels, comportement produit par une humeur corporelle, etc.) commune à la mienne. Dans l'analogisme, son intériorité et sa physicalité diffèrent de la mienne. Dans l'animisme, autrui partage avec moi une intériorité commune, mais non une même physicalité. Dans le naturalisme, lui et moi disposons d'une même physicalité, mais non d'une même intériorité. Descola Philippe, Par-delà nature et culture, Paris, Gallimard, 2015.

26. Hyde Daniel C., "Two systems of non-symbolic numerical cognition ", Frontiers in Human Neuroscience [En ligne], vol.5, mis en ligne le 29 novembre 2011, consulté le 4 février 2019, https://doi.org/10.3389/fnhum.2011.00150; Dehaene Stanislas, La Bosse des maths, Paris, Odile Jacob, 2010 ; Reznikova Zhanna \& Boris Ryabko, « Numerical competence in animals, with an insight from ants », Behaviour [En ligne], 148(4), p. 408-434, mis en ligne en 2011, consulté le 14 janvier 2018, DOI:10.1163/000579511X568562.

27. Izard Véronique \& Stanislas Dehaene, "Calibrating the mental number line », Cognition [En ligne], vol. 106, no 3, p. 1221-1247, mis en ligne en 2008, consulté le 4 février 2019, https:// doi.org/10.1016/j.cognition.2007.06.004.

28. Ifrah Georges, Histoire universelle des chiffres. Tome I. Paris, Robert Laffont, 1994, p. 46-49.

29. Miravete Sébastien, André Tricot, Kalyuga Slava \& Franck Amadieu, «Configured-group hypothesis: fast comparison of exact large quantities without counting ", Cognitive Processing, International Quarterly of Cognitive Science, vol. 18, New York, Springer, 2017, p. 447-459.

30. Les données issues de l'ethnologie expérimentale suggèrent que des sociétés possédant très peu de mots pour désigner des nombres d'objets ("un », "deux» et "beaucoup ») peuvent néanmoins comparer avec précision deux quantités d'objets en les alignant l'une contre l'autre (::::::::). Frank Michael C., Fedorenko Evelina, Lai Peter, Saxe Rebecca \& Edward Gibson, « Verbal interference suppresses exact numerical representation », Cognitive Psychology [En ligne], vol. 64, Issue 1-2, p. 74-92, mis en ligne en 2012, consulté le 5 février 2019, https://doi.org/10.1016/ j.cogpsych.2011.10.004.

31. Ifrah Georges, Histoire universelle des chiffres, op. cit.p. 164.

32. Il faut un temps considérable pour former par exemple deux rangées de 64 objets. Il suffit que les objets soient de taille importante (les navires d'une flotte) ou mobiles (du bétail) pour que cela devienne matériellement très difficile, voire impossible. Le comptage d'un en un, dans une société ne connaissant pas les groupements configurés, semble limité, quant à lui, à une trentaine d'objets - aucune société ne développe un système symbolique semblable au système décimal sans passer par la représentation de quantités sous forme de groupements configurés.

33. Ifrah Georges, Histoire universelle des chiffres. Tome I. op. cit., p. 189-191.

34. Le «transindividuel» désigne, chez Simondon, le type de relation qu'entretiennent les individus lorsque ces dernières ne se réduisent pas à de l'« interindividuel ». L'interindividuel renvoie à des relations qui ne favorisent pas la restructuration des individus, mais qui au contraire les laissent sous le contrôle de certaines structures analytiques (psychologiques, sociales, etc.). «Le transindividuel fait intervenir les sujets en tant qu'ils portent une charge de réalité pré-individuelle » (Barthélémy Jean-Hugues, « Glossaire Simondon : les 50 grandes entrées dans l'œuvre », Appareil [En ligne], 16 |2015, mis en ligne le 9 février 2016, consulté le 5 février 
2019. URL : http://journals.openedition.org/appareil/2253, DOI : 10.4000/appareil.2253). Autrement dit, une interdisciplinarité transindividuelle doit permettre aux différentes disciplines de se co-restructurer et de ne pas s'enfermer dans une même approche des phénomènes ou dans de simples échanges institutionnels.

35. Il ne s'agit jamais pour une discipline d'adopter définitivement les méthodes et les résultats d'une autre discipline, mais de se servir de certaines méthodes ou de certains résultats pour résoudre certains problèmes locaux - une discipline n'a pas besoin des autres disciplines en toute circonstance.

36. «Dans cette hypothèse, l'opposition de la culture et de la nature ne serait ni une donnée primitive, ni un aspect objectif de l'ordre du monde. On devrait voir en elle une création artificielle de la culture, un ouvrage défensif que celle-ci aurait creusé sur son pourtour parce qu'elle ne se sentait capable d'affirmer son originalité qu'en coupant tous les passages propres à attester sa connivence naturelle avec les autres manifestations de la vie. », Lévi-Strauss Claude, Les Structures élémentaires de la parenté, Paris, EHESS, 2017, p. XVI-XVII

37. Ibid., p. XVI.

38. Simondon parle de " relation » entre un " champ métastable " (les relations instables) et un "germe » (les contraintes), ou encore entre une "condition énergétique » et "structurale ", L'Individuation à la lumière des notions de forme et d'information, Grenoble, Éditions Millon, 2005, p. 44-45.

39. Ces auteurs rappellent en effet qu'un organisme vivant est sans cesse en voie de restructuration. Ils introduisent les concepts de «principe de variation" (capable de déséquilibrer une ancienne structure structurée) et de "principe d'organisation " (un ensemble de contraintes susceptibles de favoriser un nouvel équilibre). Ceux-ci sont, de toute évidence, très proches de nos concepts de relations instables et de contraintes ou encore des concepts simondoniens de métastabilité et de germe. Montévil Maël, Longo Giuseppe \& Ana Soto, «Du siècle du gène à celui de l'organisme : introduction à de nouvelles perspectives théoriques ", dans Gaudin Thierry, Lacroix Dominique, Maurel Marie-Christine \& Jean-Charles Pomerol (dir.), Sciences de la vie, sciences de l'information (Colloque de Cerisy), Paris, ISTE-Éditions, 2017, p. 9-10.

40. Naess Arne, "The shallow and the deep, long-range ecology movement. A summary", Inquiry, [en ligne], vol. 16, Issue 1, p. 95-100, mis en ligne le 29 aout 2008, consulté le 5 février 2019, https://doi.org/10.1080/00201747308601682.

41. Barthélémy Jean-Hugues, Simondon, Paris, Les Belles Lettres, 2014, p. 205-206.

42. Simondon Gilles, L'Individuation à la lumière des notions de forme et d'information, op. cit., p. 559.

43. Manzo Gianluca, «Actions, interactions et structure dans l'émergence de la stratification sociale des diplômes : un modèle de choix discrets avec externalités ", Mathématiques et sciences humaines [En ligne], 175 | Automne 2006, mis en ligne le 11 décembre 2006, consulté le 5 février 2019. URL : http://journals.openedition.org/msh/3549

44. C'est pourquoi nous travaillons sur le développement d'une mathématique plus "philosophique» pour le moment et surtout susceptible de concilier le nombre et la temporalité ; cf. Miravete Sébastien, «La durée bergsonienne comme nombre spécial » dans Worms Frédéric (dir.), Annales bergsoniennes V, Paris, Puf, 2012.

45. Miravete Sébastien, « Tetsugaku to Ninchi-shinrigaku wo Teigi suru: Berukuson Tetsugaku no Chikaku Riron to Burūnā no Ninchi-shinrigaku no Bunseki wo Tōshite » [« Définir la philosophie et la psychologie cognitive par l'analyse des théories de la perception issues de la philosophie de Bergson et de la psychologie cognitive de Bruner »], dans Hirai Y., Fujita H. \& S. Abiko (eds.), Shusuke Yamane. Berukuson Busshitsu to Kioku wo Kaibou suru: Gendai Chikaku Riron, Jikan-ron, Kokoro no Tetsugaku tono Setsuzoku [L'anatomie de Matière et mémoire de Bergson : connexions avec les théories contemporaines de la perception, de l'esprit et du temps], Tokyo, Soshi Shinsui, 2016. Texte traduit en japonais par Shusuke Yamane. 
46. La science des relations génétiques n'est pas en ce sens réductible à une pure mathématisation de la nature ou une simple objectivation de celle-ci. Sur les limites d'une approche exclusivement scientifique de la réalité, voir la très intéressante discussion des thèses de Quentin Meillassoux par Pierre Montebello dans Métaphysiques cosmomorphes - La fin du monde humain, Dijon, Les presses du réel, 2015.

47. Étienne Balibar mentionne, entre autres, les concepts fondamentaux de Chose (Lyotard) et de dissémination (Derrida) («Le structuralisme : une destitution du sujet?», Revue de métaphysique et de morale, $\mathrm{n}^{\circ} 45$, Paris, Puf, 2005, p. 15). La Chose, au même titre que le je-ne-sais-quoi chez Jankélévitch, est en effet semblable à une occurrence (cf. Miravete Sébastien, "Le postbergsonisme de Deleuze et Lyotard», dans Enaudeau C. \& F. Fruteau De Laclos, Différence, différend: Deleuze et Lyotard. Paris, Encore marine, 2015). Elle est événementielle dans la mesure où elle surgit sans être l'effet ou l'élément d'une structure. Lyotard insiste sur la nécessité de ne pas relier la Chose à d'autres éléments et Jankélévitch soutient que le je-ne-sais-quoi échappe aux sciences (cf. Miravete Sébastien, «Bergson, Jankélévitch: une opposition sur le concept de temps » dans F. Bastiani. (dir.) Bergson, Jankélévitch et Levinas. Paris, Manucius, 2017) et demeure pourtant le plus important pour l'individu - cet instant crucial où l'âme entière du sujet s'actualise.

48. Nous songeons en particulier à des auteurs comme Saussure, Piaget, Bourdieu, Deleuze, Simondon, Petitot, etc. Nous tâcherons d'éclairer dans de futures travaux dans quelle mesure ils pourraient être qualifiés, chacun à leur manière, de « (post-)structuralistes ».

\section{ABSTRACTS}

L'objectif de ce travail est de reposer un problème caractéristique des sciences humaines contemporaines. Les structuralistes sont en effet parvenus à mettre au jour des "structures ", mais ils n'ont pas expliqué la formation de celles-ci ou la manière dont celles-ci se manifestent, par exemple, sous la forme de normes phonologiques ou sociales particulières. Il ne semble pas toujours possible en effet d'expliquer leur apparition à partir du génome humain. Comment les êtres humains parviennent-ils, dès lors, à acquérir des structures et à leur donner une apparence spécifique ? Il est possible, selon nous, d'adopter une méthodologie structuraliste pour penser la genèse et la manifestation des structures, même si une telle approche peut sembler paradoxale au premier abord. Cette nouvelle manière de penser les structures diffère radicalement du structuralisme classique (1.1. à 1.4.) hérité de Jakobson et de Lévi-Strauss, dans la mesure où elle aboutit à une refonte intégrale du concept de structure (2.1. à 2.3.). Nous verrons dans quelle mesure ce (post-)structuralisme, inspiré de nos propres recherches en psychologie cognitive sur le conditionnement (2.1.) et sur les groupements configurés (2.2.), rend compte de l'apparition des structures acquises par l'être humain, mais aussi de leur nombre relativement restreint. Les notions de relation (cf. 1.3.), de sujet (cf. 2.1.), d'interdisciplinarité et d'écologie (cf. 2.3.) seront revisitées et serviront à illustrer quelques-unes des perspectives offertes en sciences humaines et sociales par un tel cadre d'investigation. Nous tâcherons enfin d'exposer dans quelle mesure un tel projet repense le rapport entre les sciences et la philosophie et renoue, en partie, avec celui proposé par Simondon dans l'allagmatique. (cf. 2.3.4.). 
INDEX

Mots-clés: structuralisme, poststructuralisme, psychologie cognitive, interdisciplinarité, Simondon, sciences humaines et sociales

\section{AUTHOR}

\section{SÉBASTIEN MIRAVETE}

Docteur en philosophie, laboratoire ERRAPHIS ; université Toulouse II ; Docteur en psychologie, laboratoire CLLE-LTC université Toulouse II ; sebastien.miravete@univ-tlse2.fr 